\title{
New monotone measure-based integrals inspired by scientific impact problem
}

\author{
Michał Boczek $^{\mathrm{a}, *}$, Anton Hovana ${ }^{\mathrm{b}}$, Ondrej Hutník ${ }^{\mathrm{b}}$, Marek Kaluszka ${ }^{\mathrm{a}, \dagger}$ \\ ${ }^{a}$ Institute of Mathematics, Lodz University of Technology, 90-924 Lodz, Poland \\ ${ }^{b}$ Institute of Mathematics, Pavol Jozef Šafárik University in Košice, 040-01 Košice, Slovakia
}

\begin{abstract}
In this paper, we define new functionals generalizing scientometric indices proposed by Mesiar and Gaggolewski in 2016 to overcome some limitations of $h$-index. These functionals are integrals with respect to a monotone measure as well as aggregation functions under some mild conditions. We derive numerous properties of the new integrals and analyze subadditivity property in detail. We also give a partial solution to the problem posed by Mesiar and Stupňanová to find an algorithm for computing the pseudo-decomposition integral of $n$-th order based on operations $\oplus=+$ and $\odot=\wedge$, which will be useful in multi-criteria decision problems.
\end{abstract}

Keywords: scientometrics; Multiple criteria analysis; $h$-index; Aggregation function; Pseudo-decomposition integral.

\section{Introduction}

In order to compare the efficiency of work of two researchers, one must construct a rule that is the most objective and fair. It turns out that the task is very difficult. Currently, there are many scientometric indices known in the literature. Their calculations are based on two inputs: number of publications and number of citations of each publication (measuring the quality and importance of publications). Nowadays, the most popular scientometric index is $h$-index introduced in 2005 by Hirsch [23. It is implemented in the largest scientific databases such as Scopus, or WoS. An axiomatic approach explaining the nature of $h$-index can be found in papers [8, 32, 33, 42], whereas its mathematical properties can be found in [17]. Torra and Narukawa [39] proved that $h$-index is the Sugeno integral

\footnotetext{
${ }^{*}$ Corresponding author

${ }^{\dagger} E$-mail adressess: $\quad$ michal.boczek.1@p.lodz.pl, ondrej.hutnik@upjs.sk, anton.hovana@student.upjs.sk, kaluszka@p.lodz.pl
} 
with respect to the counting measure. And because of specificity of the Sugeno integral, $h$-index has some drawbacks, see [31]. For example, let's consider two researchers possessing the same number of papers, say $n$, but each paper of the first one is cited $n$-times, and each paper of the second one is cited exactly $3 n$-times. Using the criterion of importance of author's publication and the number of quotations for each paper, one can see that the second researcher should have a higher scientometric index if both authors work in the same domain and have similar research experience measured by the years of work. However, $h$-index of both authors is the same and is equal to $n$. To overcome the above limitations, Mesiar and Gagolewski [28] have proposed two new indices based on the idea of $h$-index (for more details, see Section 5 ).

The present paper introduces and studies properties of two functionals: upper $n$-Sugeno integral and lower $n$-Sugeno integral of a measurable function with respect to a monotone measure. Both functionals are integrals in the spirit of definition introduced here. Integrals have many applications, especially in multicriteria decision theory, economy, optimalization or data classification [5, 10, 12, 14, $22,23,38,39,44$. To the best of our knowledge, there are only very few papers which describe the connection between integrals and scientometric indices, see [21, 39]. From those papers it follows that total number of citations is related to Choquet integral, $h$-index and Kosmulski $h(2)$-index are related to Sugeno integral, Kosmulski MAXPROD index is related to Shilkret integral, etc. In this paper we further show that the upper and lower $n$-Sugeno integrals generalize scientometric indices introduced by Mesiar \& Gągolewski and others, e.g. generalized Kosmulski index [13], iterated $h$-index [18], $\mathrm{H}_{\alpha}$ and $\mathrm{H}^{\beta}$ indices [24].

The second main result of the work is a relation between the lower $n$-Sugeno integral and some special pseudo-decomposition integral introduced by Mesiar and Stupňanová in [30] (see Theorem 4.10 below). Their question from [30, Conclusion] motivated us to describe an algorithm for computation of the pseudo-decomposition integral.

Our paper is organized as follows. In Section 2 we provide basic notations and definitions we work with. In Sections 3 and 4 we introduce new concepts of upper and lower $n$-Sugeno integral, examine their basic properties and provide their equivalent forms. Section 5 includes applications of the obtained results mainly to aggregation and scientometrics. For better readability we postpone some technical proofs of our statements to Appendix.

\section{Basic notations and preliminaries}

Let $(X, \mathcal{A})$ be a measurable space, where $\mathcal{A}$ is a $\sigma$-algebra of subsets of a non-empty set $X$. The class

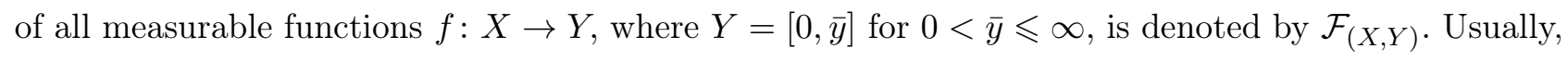


we take $\bar{y}=1$ or $\bar{y}=\infty$. A monotone measure on $\mathcal{A}$ is a nondecreasing set function $\mu: \mathcal{A} \rightarrow[0, \infty]$, i.e., $\mu(A) \leqslant \mu(B)$ whenever $A \subset B$ with $\mu(\emptyset)=0$ and $\mu(X)>0$. The range of $\mu$ we write as $\mu(\mathcal{A})$. We denote by $\mathcal{M}_{(X, \mathcal{A})}$ the class of all monotone measures on $(X, \mathcal{A})$. Given $f, g \in \mathcal{F}_{(X, Y)}$ and $\mu \in \mathcal{M}_{(X, \mathcal{A})}$, we say that $g$ dominates $f$ with respect to $\mu$ and write $f \leqslant \mu g$ if $\mu(\{f \geqslant t\}) \leqslant \mu(\{g \geqslant t\})$ for all $t$, where $\{f \geqslant t\}=\{x \in X: f(x) \geqslant t\}$. Hereafter, $a \wedge b=\min (a, b)$ and $a \vee b=\max (a, b)$. We say that a function $\circ: Y_{1} \times Y_{2} \rightarrow[0, \infty]$ is nondecreasing if $a_{1} \circ a_{2} \leqslant b_{1} \circ b_{2}$ whenever $a_{i} \leqslant b_{i}$, where $a_{i}, b_{i} \in Y_{i} \subset[0, \infty]$ for $i=1,2$.

Sugeno integral of $f \in \mathcal{F}_{(X, Y)}$ with respect to $\mu \in \mathcal{M}_{(X, \mathcal{A})}$ [37, 41] is defined by

$$
\operatorname{Su}(\mu, f):=\sup _{t \in Y}\{t \wedge \mu(\{f \geqslant t\})\} .
$$

To this day, many researchers introduced numerous generalizations of the Sugeno integral like generalized upper Sugeno integral, pseudo-decomposition integral or q-integral for $\bar{y}=\mu(X)=1$, and studied their properties [6, 7, 14, 25, 30, 36].

To make our paper as self-contained as it gets, we give some properties of the Sugeno integral that we follow later. Hereafter, $c \searrow a$ and $c \nearrow a$ means that $c \rightarrow a$ for $c>a$ and $c<a$, respectively.

Lemma 2.1. Let $(\mu, f) \in \mathcal{M}_{(X, \mathcal{A})} \times \mathcal{F}_{(X, Y)}$. The Sugeno integral possesses the following properties:

(a) $\operatorname{Su}(\mu, f) \in Y$,

(b) $t>\mu(\{f \geqslant t\})$ for $t>\operatorname{Su}(\mu, f)$ and $t<\mu(\{f \geqslant t\})$ for $t<\operatorname{Su}(\mu, f)$,

(c) $\operatorname{Su}(\mu, f)=\lim _{t>\operatorname{Su}(\mu, f)}(t \wedge \mu(\{f \geqslant t\}))$ if $\operatorname{Su}(\mu, f)>0$,

(d) $\operatorname{Su}(\mu, f)=\lim _{t \searrow \mathrm{Su}(\mu, f)}(t \vee \mu(\{f>t\}))$ if $\mathrm{Su}(\mu, f)<\bar{y}$,

(e) $\mathrm{Su}(\mu, f)=0$ if and only if $\mu(\{f \geqslant t\})=0$ for all $t>0$.

Proof. Properties (a) and (b) follow from (1), since $Y=[0, \bar{y}]$ (see also [41, Lemma 9.7]). Properties (c) and (d) follow from (b) as

$$
\begin{aligned}
& \mathrm{Su}(\mu, f)=\lim _{t \nearrow \mathrm{Su}(\mu, f)} t=\lim _{t \nearrow \operatorname{Su}(\mu, f)}(t \wedge \mu(\{f \geqslant t\})), \\
& \mathrm{Su}(\mu, f)=\lim _{t \searrow \mathrm{Su}(\mu, f)} t=\lim _{t \searrow \mathrm{Su}(\mu, f)}(t \vee \mu(\{f>t\})) .
\end{aligned}
$$

To prove (e), by the definition of the Sugeno integral we have that

$$
0=\sup _{t \geqslant 0}\{t \wedge \mu(\{f \geqslant t\})\}=(0 \wedge \mu(X)) \vee \sup _{t>0}\{t \wedge \mu(\{f \geqslant t\})\} .
$$

Now it is evident that $\mu(\{f \geqslant t\})=0$ for all $t>0$.

We formulate properties which any integral should possess.

Definition 2.2. A functional $\mathrm{J}: \mathcal{M}_{(X, \mathcal{A})} \times \mathcal{F}_{(X, Y)} \rightarrow[0, \infty]$ is called an integral if 
$\left(C_{1}\right) \mathrm{J}(\mu, f) \leqslant \mathrm{J}(\mu, g)$ whenever $f \leqslant \mu g$,

$\left(C_{2}\right) \mathrm{J}(\mu, f) \leqslant \mathrm{J}(\nu, f)$ whenever $\mu(A) \leqslant \nu(A)$ for all $A \in \mathcal{A}$,

$\left(C_{3}\right) \mathrm{J}\left(\mu, a \mathbb{1}_{A}\right)=s(a, \mu(A))$ for all $a \in Y$ and $A \in \mathcal{A}$, where $s: Y \times[0, \infty] \rightarrow Y$ is a nondecreasing function such that $s(a, 0)=s(0, b)=0$ for any $a, b$.

For a fixed $\mu \in \mathcal{M}_{(X, \mathcal{A})}$, the property $\left(C_{3}\right)$ is known in the literature as $\mu$-generated property of the integral J, see [26, Definition 3.3]. Restriction to the class of monotone measures with $\mu(X)=1$ and $Y=[0,1]$ in Definition 2.2 is closely related to fuzzy integral introduced by Struk [35]. In fact, the condition $\left(C_{3}\right)$ with $s(a, 1)=a=s(1, a)$ for any $a \in[0,1]$ implies the conditions (2) and (3) in [35, Definition 1]. However, the assumption (1) from [35, Definition 1] is stronger than $\left(C_{1}\right)$. Examples of integrals of nonnegative functions with respect to monotone measures include the Choquet integral [11], Sugeno integral or generalized upper Sugeno integral (see formula (2) in [5]) under some additional restrictions.

\section{Upper $n$-Sugeno integral}

In this section, we introduce a new type of integral with respect to a monotone measure. Our motivation for doing so comes from the lower 2- $h$-index defined by Mesiar and Gągolewski (see [19)).

We say that $\circ: Y \times Y \rightarrow Y$ is an admissible fusion map if it is nondecreasing and $0 \circ a \leqslant a$ for all $a \in Y$. The most important examples for $Y=[0, \infty]$ are: the standard addition, pseudo-addition [3], the standard product, minimum, maximum or means [2]. Moreover, for $Y=[0,1]$ the examples are: boolean conjunctions such as semicopulas [1, 5, 15], copulas [16], $t$-norms, conjunctive aggregations [2] and fuzzy conjunctions [14], and other binary operations like uninorms, $t$-semiconorms or averaging aggregations [2].

Definition 3.1. Let $(\mu, f) \in \mathcal{M}_{(X, \mathcal{A})} \times \mathcal{F}_{(X, Y)}$ and $\circ$ be an admissible fusion map. For $n \geqslant 1$ the upper $n$-Sugeno integral is defined using the recurrence

$$
\operatorname{Su}_{n+1}^{\circ}(\mu, f):=\sup _{t \in Y}\left\{\left(t \circ \operatorname{Su}_{n}^{\circ}(\mu, f)\right) \wedge \mu(\{f \geqslant t\})\right\}
$$

with the initial condition $\operatorname{Su}_{1}^{\circ}(\mu, f):=\mathrm{Su}(\mu, f)$.

Lemma 2.1(a) yields $\operatorname{Su}_{1}^{\circ}(\mu, f) \in Y$. The induction implies that $\operatorname{Su}_{n}^{\circ}(\mu, f) \in Y$ for all $n \geqslant 2$, so the functional $\mathrm{Su}_{n}^{\circ}(\mu, f)$ in Definition 3.1 is well-defined. We show that the upper $n$-Sugeno integral is an integral in the sense of Definition 2.2 .

Proposition 3.2. Let $n \geqslant 1, f, g \in \mathcal{F}_{(X, Y)}$ and $\mu, \nu \in \mathcal{M}_{(X, \mathcal{A})}$. Then

(a) $\mathrm{Su}_{n}^{\circ}(\mu, f) \leqslant \mathrm{Su}_{n}^{\circ}(\mu, g)$ whenever $f \leqslant \mu g$. 
(b) $\mathrm{Su}_{n}^{\circ}(\mu, f) \leqslant \mathrm{Su}_{n}^{\circ}(\nu, f)$ whenever $\mu(A) \leqslant \nu(A)$ for all $A \in \mathcal{A}$.

(c) $\operatorname{Su}_{n}^{\circ}\left(\mu, a \mathbb{1}_{A}\right)=s_{n}(a, \mu(A))$ for $a \in Y$ and $A \in \mathcal{A}$, where $s_{n}: Y \times[0, \infty] \rightarrow Y$ is a nondecreasing function such that $s_{n}(a, 0)=s_{n}(0, b)=0$ and $s_{n+1}(a, b)=\left(a \circ s_{n}(a, b)\right) \wedge b$ for all $a, b$.

Proof. Properties $(a)$ and $(b)$ follow immediately from Definition 3.1 and monotonicity of o. We shall prove by induction that $(c)$ holds. Clearly, $\operatorname{Su}\left(\mu, a \mathbb{1}_{A}\right)=a \wedge b$ with $b=\mu(A)$. If (c) is true for some $n \geqslant 1$, by monotonicity of function $t \mapsto t \circ b$ we have

$$
\begin{aligned}
\mathrm{Su}_{n+1}^{\circ}\left(\mu, a \mathbb{1}_{A}\right) & =\left[\left(0 \circ \mathrm{Su}_{n}^{\circ}\left(\mu, a \mathbb{1}_{A}\right)\right) \wedge \mu(X)\right] \vee \sup _{t \in(0, \bar{y}]}\left\{\left(t \circ \mathrm{Su}_{n}^{\circ}\left(\mu, a \mathbb{1}_{A}\right)\right) \wedge \mu\left(\left\{a \mathbb{1}_{A} \geqslant t\right\}\right)\right\} \\
& =\left(0 \circ s_{n}(a, b)\right) \vee\left[\left(a \circ s_{n}(a, b)\right) \wedge b\right] \\
& =\left(a \circ s_{n}(a, b)\right) \wedge b=s_{n+1}(a, b),
\end{aligned}
$$

as $0 \circ s_{n}(a, b) \leqslant s_{n}(a, b)=\mathrm{Su}_{n}^{\circ}\left(\mu, a \mathbb{1}_{A}\right) \leqslant b \leqslant \mu(X)$ and $0 \circ s_{n}(a, b) \leqslant a \circ s_{n}(a, b)$. The induction hypothesis implies that $s_{n+1}$ is nondecreasing and $s_{n+1}(a, 0)=s_{n+1}(0, b)=0$, as desired.

Now, we provide other properties of the upper $n$-Sugeno integral. From now on, to shorten the notation, we write $\mathrm{Su}_{n}^{\circ}(f)$ and $\mathrm{Su}(f)$ instead of $\mathrm{Su}_{n}^{\circ}(\mu, f)$ and $\mathrm{Su}(\mu, f)$, respectively $\mathrm{f}$ there is no ambiguity.

Proposition 3.3. (a) Let $(\mu, f) \in \mathcal{M}_{(X, \mathcal{A})} \times \mathcal{F}_{(X, Y)}$. If $\mathrm{Su}(f)=0$, then $\mathrm{Su}_{n}^{\circ}(f)=0$ for all $n$. Moreover, if $\mathrm{Su}_{k}^{\circ}(f)=0$ for some $k>1$ and $a \circ b>0$ for all $a, b>0$, then $\operatorname{Su}_{n}^{\circ}(f)=0$ for any $n$.

(b) $\mathrm{Su}_{n}^{\circ}(a f) \leqslant a \mathrm{Su}_{n}^{\circ}(f)$ for some $a>1$ and for all $(\mu, a f) \in \mathcal{M}_{(X, \mathcal{A})} \times \mathcal{F}_{(X, Y)}$ and $n \geqslant 1$ provided that $(a x) \circ(a y) \leqslant a(x \circ y)$ for all ax, ay $\in Y$. Moreover, $\mathrm{Su}_{n}^{\circ}(a f) \geqslant a \mathrm{Su}_{n}^{\circ}(f)$ for some $a \in(0,1)$ and all $(\mu, f) \in \mathcal{M}_{(X, \mathcal{A})} \times \mathcal{F}_{(X, Y)}$ whenever $(a x) \circ(a y) \geqslant a(x \circ y)$ for all $x, y \in Y$.

(c) $\mu(A)=\operatorname{Su}_{n}^{\circ}\left(\bar{y} \mathbb{1}_{A}\right)$ for any $A \in \mathcal{A}$ and $n \geqslant 1$ whenever $\mu(X) \leqslant \bar{y}$ and $\bar{y} \circ b \geqslant b$ for all $b \in Y$.

(d) (Idempotency) $\mathrm{Su}_{n}^{\circ}\left(a \mathbb{1}_{X}\right)=a$ for all $a \in Y$ and $n \geqslant 1$ if and only if $\mu(X) \geqslant \bar{y}$ and $a \circ a=a$ for any $a \in Y$.

Proof. (a) If $\mathrm{Su}(f)=0$, then by Lemma 2.1.(e) we have $\mu(\{f \geqslant t\})=0$ for all $t>0$. Hence

$$
\mathrm{Su}_{2}^{\circ}(f)=[(0 \circ 0) \wedge \mu(X)] \vee \sup _{t \in(0, \bar{y}]}\{(t \circ 0) \wedge 0\}=0
$$

as $0 \circ 0=0$. Applying the induction, we will prove that $\mathrm{Su}_{n}^{\circ}(f)=0$ for all $n$.

Assume that $\mathrm{Su}_{k}^{\circ}(f)=0$ for some $k>1$. Thus $t \circ \mathrm{Su}_{k-1}^{\circ}(f)=0$ for all $t>0$ or $\mu(\{f \geqslant t\})=0$ for all $t>0$. Suppose that $\mathrm{Su}_{k-1}^{\circ}(f)>0$. Then by the assumption on $\circ$, we have $\mu(\{f \geqslant t\})=0$ for all $t>0$, so $\mathrm{Su}(f)=0$, which implies that $\mathrm{Su}_{k-1}^{\circ}(f)=0$, a contradiction. Therefore $\mathrm{Su}_{k-1}^{\circ}(f)=0$, which leads to $\operatorname{Su}(f)=0$, and so $\mathrm{Su}_{n}^{\circ}(f)=0$ for all $n$. 
The proof of part (b) is again by induction on $n$. Clearly,

$$
\begin{aligned}
\operatorname{Su}(a f) & =\sup _{t \in Y}\{(a(t / a)) \wedge \mu(\{f \geqslant t / a\})\} \\
& \leqslant \sup _{s \leqslant \bar{y} / a}\{(a s) \wedge(a \mu(\{f \geqslant s\}))\}=a \operatorname{Su}(f)
\end{aligned}
$$

for $a>1$. Assume that the assertion holds for some $n \geqslant 1$. Then, by induction hypothesis,

$$
\begin{aligned}
\mathrm{Su}_{n+1}^{\circ}(a f) & =\sup _{s \leqslant \bar{y} / a}\left\{\left((a s) \circ \mathrm{Su}_{n}^{\circ}(a f)\right) \wedge \mu(\{f \geqslant s\})\right\} \\
& \leqslant \sup _{s \leqslant \bar{y} / a}\left\{\left((a s) \circ\left(a \mathrm{Su}_{n}^{\circ}(f)\right)\right) \wedge(a \mu(\{f \geqslant s\}))\right\} \\
& \leqslant a \sup _{s \leqslant \bar{y} / a}\left\{\left(s \circ \mathrm{Su}_{n}^{\circ}(f)\right) \wedge \mu(\{f \geqslant s\})\right\}=a \mathrm{Su}_{n+1}^{\circ}(f) .
\end{aligned}
$$

The proof for the case $0<a<1$ is analogous. To prove (c) and (d) one can use Proposition 3.2 (c).

Proposition 3.4. The sequence $\left(\mathrm{Su}_{n}^{\circ}(f)\right)_{n \geqslant 1}$ is nondecreasing for all $(\mu, f) \in \mathcal{M}_{(X, \mathcal{A})} \times \mathcal{F}_{(X, Y)}$ if and only if $a \circ b \geqslant a \wedge b$ for all $a, b \in Y$.

Proof. " $\Rightarrow$ " By Proposition 3.2 (c) we get $\mathrm{Su}_{2}^{\circ}\left(a \mathbb{1}_{A}\right)=(a \circ(a \wedge b)) \wedge b$, where $A \in \mathcal{A}, a \in Y$ and $b=\mu(A)$. Since $\operatorname{Su}_{2}^{\circ}\left(a \mathbb{1}_{A}\right) \geqslant \operatorname{Su}_{1}^{\circ}\left(a \mathbb{1}_{A}\right)$, we have $(a \circ(a \wedge b)) \wedge b \geqslant a \wedge b$ for all $a \in Y, b \in \mu(\mathcal{A}) \cap Y$ and any monotone measure $\mu$. Hence, $a \circ b \geqslant(a \circ b) \wedge b \geqslant(a \circ(a \wedge b)) \wedge b \geqslant a \wedge b$ for all $a, b \in Y$. " $\Leftarrow$ " By assumption $\operatorname{Su}_{2}^{\circ}(f) \geqslant \sup _{t \in Y}\{(t \wedge \mathrm{Su}(f)) \wedge \mu(\{f \geqslant t\})\}=\operatorname{Su}(f)$. Suppose that $\operatorname{Su}_{n}^{\circ}(f) \geqslant \operatorname{Su}_{n-1}^{\circ}(f)$ for some $n>1$. By the monotonicity $t \mapsto a \circ t$ for all $a$ and the induction hypothesis, we obtain

$$
\begin{aligned}
\mathrm{Su}_{n+1}^{\circ}(f) & =\sup _{t \in Y}\left\{\left(t \circ \mathrm{Su}_{n}^{\circ}(f)\right) \wedge \mu(\{f \geqslant t\})\right\} \\
& \geqslant \sup _{t \in Y}\left\{\left(t \circ \mathrm{Su}_{n-1}^{\circ}(f)\right) \wedge \mu(\{f \geqslant t\})\right\}=\mathrm{Su}_{n}^{\circ}(f),
\end{aligned}
$$

thus $\left(\mathrm{Su}_{n}^{\circ}(f)\right)_{n \geqslant 1}$ is a nondecreasing sequence.

Let $(\mu, f) \in \mathcal{M}_{(X, \mathcal{A})} \times \mathcal{F}_{(X, Y)}$. Recall that

$$
\operatorname{Su}(f)=\inf _{t \in Y}\{t \vee \mu(\{f>t\})\}=\sup _{A \in \mathcal{A}}\left\{\inf _{x \in A} f(x) \wedge \mu(A)\right\}
$$

see [5, 37, 41]. We present formulas for the upper $n$-Sugeno integral, which have the forms as in (2).

Theorem 3.5. Let $\circ$ be an admissible fusion map that is continuous in the first argument. Then for all $(\mu, f) \in \mathcal{M}_{(X, \mathcal{A})} \times \mathcal{F}_{(X, Y)}$ and $n \geqslant 2$ we have

$$
\mathrm{Su}_{n}^{\circ}(f)=\inf _{t \in Y}\left\{\left(t \circ \mathrm{Su}_{n-1}^{\circ}(f)\right) \vee \mu(\{f>t\})\right\} .
$$

Proof. The proof is given in Appendix. 
Theorem 3.6. For all $(\mu, f) \in \mathcal{M}_{(X, \mathcal{A})} \times \mathcal{F}_{(X, Y)}$ and $n \geqslant 2$ we have

$$
\mathrm{Su}_{n}^{\circ}(f)=\sup _{A \in \mathcal{A}}\left\{\left(\inf _{x \in A} f(x) \circ \mathrm{Su}_{n-1}^{\circ}(f)\right) \wedge \mu(A)\right\} .
$$

Proof. Arguing as in the proof of Theorem 2.2 in [7], we shall show more than it is needed, i.e., if a map $\circ$ is nondecreasing in the first coordinate, then

$$
\sup _{t \in Y}\{(t \circ a) \wedge \mu(\{f \geqslant t\})\}=\sup _{A \in \mathcal{A}}\left\{\left(\inf _{x \in A} f(x) \circ a\right) \wedge \mu(A)\right\}
$$

for all $a \in Y$. In fact, let $t \in Y$ and $A_{t}=\{f \geqslant t\}$. Thus, $\inf _{x \in A_{t}} f(x) \geqslant t$ and

$$
(t \circ a) \wedge \mu(\{f \geqslant t\}) \leqslant\left(\inf _{x \in A_{t}} f(x) \circ a\right) \wedge \mu\left(A_{t}\right) \leqslant \sup _{A \in \mathcal{A}}\left\{\left(\inf _{x \in A} f(x) \circ a\right) \wedge \mu(A)\right\} .
$$

Therefore, the left hand side in (3) is not greater than the right one. Let $A \in \mathcal{A}$ and $t_{0}=\inf _{x \in A} f(x)$. Then $A \subset\left\{f \geqslant t_{0}\right\}$ and

$$
\left(\inf _{x \in A} f(x) \circ a\right) \wedge \mu(A) \leqslant\left(t_{0} \circ a\right) \wedge \mu\left(\left\{f \geqslant t_{0}\right\}\right) \leqslant \sup _{t \in Y}\{(t \circ a) \wedge \mu(\{f \geqslant t\})\} .
$$

Thus, the left hand side in (3) is greater than or equal to the right one, which finishes the proof.

The most important property of an integral is subadditivity. The following concept was introduced in [5].

Definition 3.7. Let $\mu \in \mathcal{M}_{(X, \mathcal{A})}$. We say that $f, g \in \mathcal{F}_{(X, Y)}$ are $\mu$-subadditive with respect to a map $\nabla: \mu(\mathcal{A}) \times \mu(\mathcal{A}) \rightarrow \mu(\mathcal{A})(\mu-\nabla$-subadditive for short $)$ if

$$
\mu(\{f>a\} \cup\{g>b\}) \leqslant \mu(\{f>a\}) \nabla \mu(\{g>b\})
$$

for all $a, b \in Y$.

Note that all functions $f, g$ are $\mu$ - $\nabla$-subadditive if $a \nabla b=(a+b) \wedge \mu(X)$ whenever the monotone measure $\mu$ is subadditive, that is, $\mu(A \cup B) \leqslant \mu(A)+\mu(B)$ for all $A, B \in \mathcal{A}$. Recall that $f, g \in \mathcal{F}_{(X, Y)}$ are comonotone if $(f(x)-f(y))(g(x)-g(y)) \geqslant 0$ for all $x, y \in X$. Equivalently, $f$ and $g$ are comonotone, if for any $t \in Y$ either $\{f>t\} \subset\{g>t\}$ or $\{g>t\} \subset\{f>t\}$. Thus, comonotone functions are $\mu$ - $\nabla$-subadditive for $\nabla \geqslant \vee$. For more examples of $\mu$ - $\nabla$-subadditive functions we refer to [5].

Theorem 3.8. Assume that $\nabla: \mu(\mathcal{A}) \times \mu(\mathcal{A}) \rightarrow \mu(\mathcal{A}), f, g \in \mathcal{F}_{(X, Y)}$ and

$$
[((a+b) \wedge \bar{y}) \odot((c+d) \wedge \bar{y})] \vee(\alpha \nabla \beta) \leqslant[(a \odot c) \vee \alpha]+[(b \odot d) \vee \beta]
$$

for $a, b, c, d \in Y$ and $\alpha, \beta \in \mu(\mathcal{A})$ such that $a+b, c+d \in Y$ with $\odot \in\{\circ, \mathrm{P}\}$, where the admissible fusion map $\circ$ is continuous in the first argument and $x \mathrm{P} y=x$ for any $x, y$. If $f, g$ are $\mu-\nabla$-subadditive and $f+g \in \mathcal{F}_{(X, Y)}$, then

$$
\mathrm{Su}_{n}^{\circ}(f+g) \leqslant \mathrm{Su}_{n}^{\circ}(f)+\mathrm{Su}_{n}^{\circ}(g)
$$


for $n \geqslant 1$. Moreover, $\mu$ is a subadditive monotone measure whenever there is some $n \geqslant 1$ such that (5) holds for all $f, g \in \mathcal{F}_{(X, Y)}$ with $\mu(X) \leqslant \bar{y}$ and $\bar{y} \circ b \geqslant b$ for all $b \in Y$.

Proof. The proof is by induction on $n$. Let $n=1$. Evidently, $\{f \leqslant a\} \cap\{g \leqslant b\} \subset\{f+g \leqslant a+b\}$. As $f(x)+g(x) \leqslant \bar{y}$ for any $x$ and $f, g$ are $\mu$ - $\nabla$-subadditive, we have

$$
\begin{aligned}
{[(a+b) \wedge \bar{y}] \vee \mu(\{f+g>(a+b) \wedge \bar{y}\}) } & \leqslant[(a+b) \wedge \bar{y}] \vee[\mu(\{f>a\}) \nabla \mu(\{g>b\})] \\
& \leqslant[a \vee \mu(\{f>a\})]+[b \vee \mu(\{g>b\})],
\end{aligned}
$$

where the last inequality holds by (4) with $x \odot y=x$. By (2) we get

$$
\mathrm{Su}(f+g) \leqslant[a \vee \mu(\{f>a\})]+[b \vee \mu(\{g>b\})]
$$

Taking the lower bound for $a, b \in Y$ finishes the proof of (5) for $n=1$.

Assume that (5) holds for some $n>1$. By $\mu$ - $\nabla$-subadditivity and (4) with $\odot=\circ$, we get

$$
\begin{aligned}
{[[(a+b) \wedge \bar{y}] \circ[} & \left.\left.\left(\mathrm{Su}_{n}^{\circ}(f)+\mathrm{Su}_{n}^{\circ}(g)\right) \wedge \bar{y}\right]\right] \vee \mu(\{f+g>(a+b) \wedge \bar{y}\}) \\
& \leqslant\left[[(a+b) \wedge \bar{y}] \circ\left[\left(\mathrm{Su}_{n}^{\circ}(f)+\mathrm{Su}_{n}^{\circ}(g)\right) \wedge \bar{y}\right]\right] \vee[\mu(\{f>a\}) \nabla \mu(\{g>b\})] \\
& \leqslant\left[\left(a \circ \mathrm{Su}_{n}^{\circ}(f)\right) \vee \mu(\{f>a\})\right]+\left[\left(b \circ \mathrm{Su}_{n}^{\circ}(g)\right) \vee \mu(\{g>b\})\right] .
\end{aligned}
$$

By the induction hypothesis and $\operatorname{Su}_{n}^{\circ}(f+g) \leqslant \bar{y}$, we have for all $a, b \in Y$

$$
\begin{aligned}
{\left[[(a+b) \wedge \bar{y}] \circ \mathrm{Su}_{n}^{\circ}(f+g)\right] } & \vee \mu(\{f+g>(a+b) \wedge \bar{y}\}) \\
& \leqslant\left[\left(a \circ \operatorname{Su}_{n}^{\circ}(f)\right) \vee \mu(\{f>a\})\right]+\left[\left(b \circ \operatorname{Su}_{n}^{\circ}(g)\right) \vee \mu(\{g>b\})\right] .
\end{aligned}
$$

As a consequence of Theorem 3.5 we obtain

$$
\mathrm{Su}_{n+1}^{\circ}(f+g) \leqslant\left[\left(a \circ \mathrm{Su}_{n}^{\circ}(f)\right) \vee \mu(\{f>a\})\right]+\left[\left(b \circ \mathrm{Su}_{n}^{\circ}(g)\right) \vee \mu(\{g>b\})\right] .
$$

Taking the lower bound for $a$ and then for $b$ we finish the proof of (5).

Suppose that (5) is satisfied for some $n \geqslant 1$. Then Proposition 3.3 (c) yields $\operatorname{Su}_{k}^{\circ}\left(\bar{y} \mathbb{1}_{D}\right)=\mu(D)$ for all $D$ and $k$. Putting $f=\bar{y} \mathbb{1}_{A}$ and $g=\bar{y} \mathbb{1}_{B \backslash A}$ in (5), we obtain $\mu(A \cup B) \leqslant \mu(A)+\mu(B \backslash A) \leqslant \mu(A)+\mu(B)$. This completes the proof.

Corollary 3.9. If $\mu \in \mathcal{M}_{(X, \mathcal{A})}$ is subadditive and $\mu(X) \leqslant \bar{y}$, then

$$
\mathrm{Su}_{n}^{+}(f+g) \leqslant \mathrm{Su}_{n}^{+}(f)+\mathrm{Su}_{n}^{+}(g)
$$

for any $n \geqslant 1$ and all $f, g \in \mathcal{F}_{(X, Y)}$ such that $f+g \in \mathcal{F}_{(X, Y)}$. Moreover, if there is $n$ such that (6) holds for all $f, g \in \mathcal{F}_{(X, Y)}$, then $\mu$ is a subadditive monotone measure. 
Proof. The assertion follows from Theorem 3.8 for $a \circ b=(a+b) \wedge \bar{y}$ and $a \nabla b=(a+b) \wedge \mu(X)$.

Corollary 3.10. If $f, g \in \mathcal{F}_{(X, Y)}$ are comonotone functions and $\mu(\mathcal{A}) \subset Y$, then $\operatorname{Su}_{n}^{+}(f+g) \leqslant$ $\mathrm{Su}_{n}^{+}(f)+\mathrm{Su}_{n}^{+}(g)$ for all $n \geqslant 1$ and $f+g \in \mathcal{F}_{(X, Y)}$.

Proof. Apply Theorem 3.8 for $a \circ b=(a+b) \wedge \bar{y}$ and $\nabla=\vee$.

Condition (4) is valid if $\nabla \leqslant+$ and $[(a+b) \wedge \bar{y}] \circ[(c+d) \wedge \bar{y}] \leqslant(a \circ c)+(b \circ d)$ for any $a, b, c, d \in Y$. Examples of maps o satisfying the last inequality are:

(i) $a \circ b=\lambda a$ for $Y=[0,1]$ and $\lambda \in(0,1]$, or $Y=[0, \infty]$ and $\lambda>0$,

(ii) $a \circ b=a^{\gamma}$ with $\gamma \in(0,1)$,

(iii) $a \circ b=\lambda(a+b)+(1-\lambda)(a \vee b)$ for $\lambda \in[0,1]$ and $Y=[0, \infty]$,

(iv) $a \circ b=a+b-a b$ for $Y=[0,1]$.

As we have shown above, the upper $n$-Sugeno integral possesses several properties of the Sugeno integral, but not all. Hereafter, $a \mathbb{1}_{X} \vee f=\left(a \mathbb{1}_{X}\right) \vee f$ and $a \mathbb{1}_{X} \wedge f=\left(a \mathbb{1}_{X}\right) \wedge f$. We say that the integral $\mathrm{J}$ is maxitive homogeneous and minitive homogeneous if $\mathrm{J}\left(\mu, a \mathbb{1}_{X} \vee f\right)=a \vee \mathrm{J}(\mu, f)$ and $\mathrm{J}\left(\mu, a \mathbb{1}_{X} \wedge f\right)=a \wedge \mathrm{J}(\mu, f)$ for any $a, \mu, f$, respectively. Next example demonstrates that it is not the case of upper and lower $n$-Sugeno integral.

Example 3.11. Let $f=0.25 \mathbb{1}_{A}+0.75 \mathbb{1}_{B}$, where $A \cap B=\emptyset$ and $A \cup B=X$. Assume that $\mu(X)=1$ and $\mu(B)=0.5$. It is clear that $(1 / 3) \mathbb{1}_{X} \wedge f=0.25 \mathbb{1}_{A}+(1 / 3) \mathbb{1}_{B}$ and $(1 / 3) \mathbb{1}_{X} \vee f=(1 / 3) \mathbb{1}_{A}+0.75 \mathbb{1}_{B}$. Thus,

$$
\mathrm{Su}_{2}^{+}(f)=0.75, \quad \mathrm{Su}_{2}^{+}\left((1 / 3) \mathbb{1}_{X} \wedge f\right)=7 / 12, \quad \mathrm{Su}_{2}^{+}\left((1 / 3) \mathbb{1}_{X} \vee f\right)=5 / 6,
$$

so $\mathrm{Su}_{2}^{+}\left((1 / 3) \mathbb{1}_{X} \wedge f\right)>(1 / 3) \wedge \mathrm{Su}_{2}^{+}(f)$ and $\mathrm{Su}_{2}^{+}\left((1 / 3) \mathbb{1}_{X} \vee f\right)>(1 / 3) \vee \mathrm{Su}_{2}^{+}(f)$.

Now we give one sufficient condition for minitive/maxitive homogeneity of the integral.

Proposition 3.12. Let $n \geqslant 2$ and $(\mu, f) \in \mathcal{M}_{(X, \mathcal{A})} \times \mathcal{F}_{(X, Y)}$. Then

(a) $\operatorname{Su}_{n}^{\circ}\left(a \mathbb{1}_{X} \wedge f\right)=a \wedge \mathrm{Su}_{n}^{\circ}(f)$ for any $a \in Y$ if $(a \wedge b) \circ(a \wedge c)=a \wedge(b \circ c)$ for all $a, b, c \in Y$.

(b) $\mathrm{Su}_{n}^{\circ}\left(a \mathbb{1}_{X} \vee f\right)=a \vee \mathrm{Su}_{n}^{\circ}(f)$ for any $a \in Y$ if $(a \vee b) \circ(a \vee c)=a \vee(b \circ c)$ for all $a, b, c \in Y$.

Proof. We show only $(a)$ since the proof of $(b)$ is analogous. Since $\operatorname{Su}\left(a \mathbb{1}_{X} \wedge f\right)=a \wedge \operatorname{Su}(f)$, we have from Theorem 3.6 that $\operatorname{Su}_{2}^{\circ}\left(a \mathbb{1}_{X} \wedge f\right)=\sup _{A \in \mathcal{A}}\left\{\left[\left(a \wedge \inf _{x \in A} f(x)\right) \circ(a \wedge \operatorname{Su}(f))\right] \wedge \mu(A)\right\}=a \wedge \operatorname{Su}_{2}^{\circ}(f)$. The second induction step proceeds similarly.

The admissible fusion map $x \circ y=\varphi(x) \wedge \gamma(y)$ satisfies the condition of Proposition 3.12 (a) if $\varphi, \gamma: Y \rightarrow Y$ are nondecreasing functions such that $\varphi(a) \wedge \gamma(a)=a$ for any $a \in Y$. The assumption of Proposition 3.12 (b) holds if $x \circ y=\varphi(x) \vee \gamma(y)$, where the functions $\varphi, \gamma: Y \rightarrow Y$ are nondecreasing with $\varphi(a) \vee \gamma(a)=a$ for all $a \in Y$. 


\section{Lower $n$-Sugeno integral}

This section is devoted to defining a new functional generalizing the upper 2 - $h$-index $(18)$ and its properties. We say that $\star:[0, \infty] \times Y \rightarrow[0, \infty]$ is a link map if it is nondecreasing and $0 \star a \leqslant a$ for all $a \in Y$. Clearly, the link map coincides with the admissible fusion map if and only if $Y=[0, \infty]$.

Definition 4.1. Let $(\mu, f) \in \mathcal{M}_{(X, \mathcal{A})} \times \mathcal{F}_{(X, Y)}$ and $\star$ be a link map. For $n \geqslant 1$ the lower $n$-Sugeno integral is defined by

$$
\mathrm{Su}_{\star}^{n+1}(\mu, f):=\sup _{t \in Y}\left\{t \wedge\left(\mu(\{f \geqslant t\}) \star \mathrm{Su}_{\star}^{n}(\mu, f)\right)\right\},
$$

where $\mathrm{Su}_{\star}^{1}(\mu, f)=\operatorname{Su}(\mu, f)$.

It is clear that $\operatorname{Su}_{\star}^{n}(\mu, f) \in Y$ for all $n$. The next proposition shows that the lower $n$-Sugeno integral satisfies all the properties in Definition 2.2 .

Proposition 4.2. Let $n \geqslant 1, f, g \in \mathcal{F}_{(X, Y)}$ and $\mu, \nu \in \mathcal{M}_{(X, \mathcal{A})}$.

(a) If $f \leqslant \mu$, then $\operatorname{Su}_{\star}^{n}(\mu, f) \leqslant \operatorname{Su}_{\star}^{n}(\mu, g)$.

(b) If $\mu(A) \leqslant \nu(A)$ for all $A \in \mathcal{A}$, then $\operatorname{Su}_{\star}^{n}(\mu, f) \leqslant \mathrm{Su}_{\star}^{n}(\nu, f)$.

(c) $\operatorname{Su}_{\star}^{n}\left(\mu, a \mathbb{1}_{A}\right)=s_{n}(a, \mu(A))$ for $a \in Y$ and $A \in \mathcal{A}$, where $s_{n}: Y \times[0, \infty] \rightarrow Y$ is a nondecreasing function such that $s_{n}(a, 0)=s_{n}(0, b)=0$ and $s_{n+1}(a, b)=a \wedge\left[b \star s_{n}(a, b)\right]$ for all $a, b$.

Proof. Parts $(a)$ and $(b)$ are immediate by induction.

(c) We have the following recurrence formula

$$
\mathrm{Su}_{\star}^{n+1}\left(\mu, a \mathbb{1}_{A}\right)=\left[a \wedge\left(\mu(A) \star \mathrm{Su}_{\star}^{n}\left(\mu, a \mathbb{1}_{A}\right)\right)\right] \vee\left[\bar{y} \wedge\left(0 \star \mathrm{Su}_{\star}^{n}\left(\mu, a \mathbb{1}_{A}\right)\right)\right],
$$

where $a \in Y$. First we show that

$$
\mathrm{Su}_{\star}^{n}\left(\mu, a \mathbb{1}_{A}\right) \leqslant a
$$

for all $n$ and $a \in Y$. We use induction on $n$. In fact, $\operatorname{Su}\left(a \mathbb{1}_{A}\right)=a \wedge \mu(A) \leqslant a$. Assume that (7) holds for some $n$. Since $0 \star a \leqslant a$, we have

$$
\mathrm{Su}_{\star}^{n+1}\left(\mu, a \mathbb{1}_{A}\right) \leqslant[a \wedge(\mu(A) \star a)] \vee[\bar{y} \wedge(0 \star a)] \leqslant a \vee a=a
$$

and the proof of (7) is complete. From (7), we obtain $0 \star \operatorname{Su}_{\star}^{n+1}\left(\mu, a \mathbb{1}_{A}\right) \leqslant 0 \star a \leqslant a \leqslant \bar{y}$ for all $a \in Y$. Hence,

$$
\mathrm{Su}_{\star}^{n+1}\left(\mu, a \mathbb{1}_{A}\right)=\left[a \wedge\left(\mu(A) \star \operatorname{Su}_{\star}^{n}\left(\mu, a \mathbb{1}_{A}\right)\right)\right] \vee\left[0 \star \operatorname{Su}_{\star}^{n}\left(\mu, a \mathbb{1}_{A}\right)\right] .
$$

As $0 \star \operatorname{Su}_{\star}^{n}\left(\mu, a \mathbb{1}_{A}\right) \leqslant 0 \star a \leqslant a$ and $0 \star \operatorname{Su}_{\star}^{n}\left(\mu, a \mathbb{1}_{A}\right) \leqslant \mu(A) \star \operatorname{Su}_{\star}^{n}\left(\mu, a \mathbb{1}_{A}\right)$, we get

$$
\mathrm{Su}_{\star}^{n+1}\left(\mu, a \mathbb{1}_{A}\right)=a \wedge\left[\mu(A) \star \mathrm{Su}_{\star}^{n}\left(\mu, a \mathbb{1}_{A}\right)\right] .
$$

Applying induction on $n$, we obtain the statement (c). 
To shorten the notation, we write $\mathrm{Su}_{\star}^{n}(f)$ instead of $\mathrm{Su}_{\star}^{n}(\mu, f)$ if there is no ambiguity. Hereafter, for a link map $\star$ and $\mu \in \mathcal{M}_{(X, \mathcal{A})}$ we use the convention $\mu_{k+1}^{\star}(A):=\mu(A) \star \mu_{k}^{\star}(A)$ for all $k \geqslant 1$ with $\mu_{1}^{\star}(A)=\mu(A)$ provided that $\mu_{k}^{\star}(X) \in Y$ for all $k$. It is evident that $\mu_{n}^{\star}$ is a monotone measure if $\mu_{n}^{\star}(X)>0$. Some properties of the lower $n$-Sugeno integral are analogous to those of the upper $n$-Sugeno integral, which is shown in what follows.

Proposition 4.3. Let $(\mu, f) \in \mathcal{M}_{(X, \mathcal{A})} \times \mathcal{F}_{(X, Y)}$ and $A \in \mathcal{A}$.

(a) If $\mathrm{Su}(f)=0$, then $\mathrm{Su}_{\star}^{n}(f)=0$ for all $n \geqslant 2$. If $\mathrm{Su}_{\star}^{k}(f)=0$ for some $k>1$ and $a \star b>0$ for all $a, b>0$, then $\operatorname{Su}_{\star}^{n}(f)=0$ for all $n \geqslant 1$.

(b) $\mathrm{Su}_{\star}^{n}(f)=\lim _{t>\mathrm{Su}_{\star}^{n}(f)}\left(t \wedge\left(\mu(\{f \geqslant t\}) \star \mathrm{Su}_{\star}^{n-1}(f)\right)\right)$ if $\mathrm{Su}_{\star}^{n-1}(f)>0$.

(c) $\mathrm{Su}_{\star}^{n}(f)=\lim _{t \searrow \mathrm{Su}_{\star}^{n}(f)}\left(t \vee\left(\mu(\{f>t\}) \star \mathrm{Su}_{\star}^{n-1}(f)\right)\right)$ if $\mathrm{Su}_{\star}^{n}(f)<\bar{y}$.

(d) There is $a>1$ such that $\operatorname{Su}_{\nu}^{n}(a g) \leqslant a \operatorname{Su}_{\nu}^{n}(g)$ for all $(\nu, a g) \in \mathcal{M}_{(X, \mathcal{A})} \times \mathcal{F}_{(X, Y)}$ and $n \geqslant 1$, if $x \star(a y) \leqslant a(x \star y)$ for all $x \in \nu(\mathcal{A})$ and ay $\in Y$. Moreover, $\operatorname{Su}_{\nu}^{n}(a g) \geqslant a \operatorname{Su}_{\nu}^{n}(g)$ for some a $\in(0,1)$ and for all $(\nu, g) \in \mathcal{M}_{(X, \mathcal{A})} \times \mathcal{F}_{(X, Y)}$ and $n \geqslant 1$, if $x \star(a y) \geqslant a(x \star y)$ for all $x \in \nu(\mathcal{A})$ and $y \in Y$.

(e) $\mathrm{Su}_{\star}^{n}\left(a \mathbb{1}_{A}\right)=\mu_{n}^{\star}(A)$ for $a \in\left[\max _{1 \leqslant k \leqslant n} \mu_{k}^{\star}(A), \bar{y}\right]$.

(f) (Idempotency) $\operatorname{Su}_{\star}^{n}\left(a \mathbb{1}_{X}\right)=a$ for all $a \in Y$ and $n \geqslant 1$ if and only if $\mu(X) \geqslant \bar{y}$ and $\mu(X) \star a \geqslant a$ for all $a \in Y$.

Proof. (a) The first assertion follows from Lemma 2.1 (e) and the induction, since $0 \star 0=0$. If $\mathrm{Su}_{\star}^{k}(f)=0$ for some $k>1$, then repeating similar argument as in the proof of Proposition 3.3 (a), we get the assertion.

(b) and $(c)$ By monotonicity of $\star$, we have $t>\mu(\{f \geqslant t\}) \star \mathrm{Su}_{\star}^{n-1}(f)$ for $t>\mathrm{Su}_{\star}^{n}(f)$ and $t<\mu(\{f \geqslant$ $t\}) \star \mathrm{Su}_{\star}^{n-1}(f)$ for $t<\mathrm{Su}_{\star}^{n}(f)$. In consequence, both properties (b) and (c) hold. See the proof of Lemma $2.1(\mathrm{c})-(\mathrm{d})$.

(d) The proof is similar to that of Proposition $3.3(\mathrm{~b})$.

$(e)$ and $(f)$ The proofs go by induction on $n$; see (8).

Proposition 4.4. The sequence $\left(\mathrm{Su}_{\star}^{n}(f)\right)_{n=1}^{\infty}$ is nondecreasing for all $(\mu, f) \in \mathcal{M}_{(X, \mathcal{A})} \times \mathcal{F}_{(X, Y)}$ if and only if $a \star b \geqslant a \wedge b$ for all $a \in[0, \infty]$ and $b \in Y$.

Proof. " $\Rightarrow$ " By (8) for $n=1$, we have $\operatorname{Su}_{\star}^{2}\left(a \mathbb{1}_{A}\right)=a \wedge[b \star(a \wedge b)]$, where $A \in \mathcal{A}, a \in Y$ and $b=\mu(A)$. Since $\operatorname{Su}_{\star}^{1}\left(a \mathbb{1}_{A}\right) \leqslant \operatorname{Su}_{\star}^{2}\left(a \mathbb{1}_{A}\right)$, we obtain $a \wedge b \leqslant a \wedge[b \star(a \wedge b)] \leqslant b \star a$ for all $a \in Y$ and $b \in[0, \infty]$, as desired.

" $\Leftarrow$ " The proof is similar to that of Proposition 3.4, so we omit it.

Theorem 4.5. For all $(\mu, f) \in \mathcal{M}_{(X, \mathcal{A})} \times \mathcal{F}_{(X, Y)}$ and $n \geqslant 2$

$$
\mathrm{Su}_{\star}^{n}(f)=\sup _{A \in \mathcal{A}}\left\{\inf _{x \in A} f(x) \wedge\left(\mu(A) \star \mathrm{Su}_{\star}^{n-1}(f)\right)\right\} .
$$


Proof. Use the same arguments as in the proof of Theorem 3.6 for a nondecreasing map $\star$ in the first coordinate.

The following extension of the first equality in $(2)$ will be needed to prove the subadditivity property of the lower integral.

Theorem 4.6. Assume that $\star$ is a continuous link map in the first argument. Then for each $(\mu, f) \in$ $\mathcal{M}_{(X, \mathcal{A})} \times \mathcal{F}_{(X, Y)}$ and $n \geqslant 2$ we have

$$
\mathrm{Su}_{\star}^{n}(f)=\inf _{t \in Y}\left\{t \vee\left(\mu(\{f>t\}) \star \mathrm{Su}_{\star}^{n-1}(f)\right)\right\} .
$$

Proof. The proof is given in Appendix.

Next, we show that the lower $n$-Sugeno integral is also a subadditive functional under some extra assumptions.

Theorem 4.7. Suppose that $\nabla: \mu(\mathcal{A}) \times \mu(\mathcal{A}) \rightarrow \mu(\mathcal{A}), f, g \in \mathcal{F}_{(X, Y)}$ and

$$
[(a+b) \wedge \bar{y}] \vee[(\alpha \nabla \beta) \circledast((c+d) \wedge \bar{y})] \leqslant[a \vee(\alpha \circledast c)]+[b \vee(\beta \circledast d)]
$$

for $a, b, c, d \in Y$ and $\alpha, \beta \in \mu(\mathcal{A})$ with $\circledast \in\{\star, P\}$, where $\star$ is a continuous link map in the first argument and $x \mathrm{P} y=x$ for any $x, y$. If $f, g$ are $\mu-\nabla$-subadditive and $f+g \in \mathcal{F}_{(X, Y)}$, then

$$
\mathrm{Su}_{\star}^{n}(f+g) \leqslant \mathrm{Su}_{\star}^{n}(f)+\mathrm{Su}_{\star}^{n}(g)
$$

for all $n \geqslant 1$. Moreover, if 10 is valid for all $f, g \in \mathcal{F}_{(X, Y)}$ such that $f+g \in \mathcal{F}_{(X, Y)}$ and $n$ such that $\mu_{n}^{\star}(X)>0$, then the monotone measure $\mu_{n}^{\star}$ is subadditive.

Proof. We use the induction by $n$. The proof of subadditivity of the Sugeno integral (the case $n=1$ and $\circledast=\mathrm{P}$ ) can be found in the proof of Theorem 3.8. Assume that inequality 10 holds for some $n \geqslant 2$ and all $\mu$ - $\nabla$-subadditive functions $f, g$. Combining inductive hypothesis and (9) with $\circledast=\star$ yields

$$
\begin{aligned}
{[(a+b) \wedge \bar{y}] \vee } & {\left[\mu(\{f+g>(a+b) \wedge \bar{y}\}) \star\left(\mathrm{Su}_{\star}^{n}(f+g) \wedge \bar{y}\right)\right] } \\
& \leqslant[(a+b) \wedge \bar{y}] \vee\left[(\mu(\{f>a\}) \nabla \mu(\{g>b\})) \star\left(\left(\mathrm{Su}_{\star}^{n}(f)+\mathrm{Su}_{\star}^{n}(g)\right) \wedge \bar{y}\right)\right] \\
& \leqslant\left[a \vee\left(\mu(\{f>a\}) \star \operatorname{Su}_{\star}^{n}(f)\right)\right]+\left[b \vee\left(\mu(\{g>b\}) \star \operatorname{Su}_{\star}^{n}(g)\right)\right]
\end{aligned}
$$

for all $a, b \in Y$. By Theorem 4.6, we get

$$
\mathrm{Su}_{\star}^{n+1}(f+g) \leqslant\left[a \vee\left(\mu(\{f>a\}) \star \mathrm{Su}_{\star}^{n}(f)\right)\right]+\left[b \vee\left(\mu(\{g>b\}) \star \mathrm{Su}_{\star}^{n}(g)\right)\right]
$$

for any $a, b \in Y$. Taking infimum over $a$ and then with $b$ gives 10 .

Put $f=a \mathbb{1}_{A}$ and $g=a \mathbb{1}_{B \backslash A}$ in 10 , where $a \geqslant \max _{k \leqslant n} \mu_{k}^{\star}(A \cup B)$. Then from Proposition 4.3(e) and by monotonicity of $\mu_{n}^{\star}$, we get $\mu_{n}^{\star}(A \cup B) \leqslant \mu_{n}^{\star}(A)+\mu_{n}^{\star}(B \backslash A) \leqslant \mu_{n}^{\star}(A)+\mu_{n}^{\star}(B)$, as desired. 
Example 4.8. There are many link maps $\star$ with $Y=[0, \infty]$ such that subadditivity of $\mu_{n}^{\star}$ with arbitrary $n$ implies subadditivity of $\mu$. For instance, all idempotent operators (e.g. $a \star b=a^{p} b^{1-p}$ and $a \star b=p(a \wedge b)+(1-p)(a \vee b)$ with $p \in(0,1))$ as well as mappings
a) $a \star b=f(a) f(b)$,
b) $a \star b=g(a b)$,
c) $a \star b=h(a+b)$,
d) $a \star b=\left(a^{q}+b^{q}\right)^{1 / q}$,

where $q>0$ and $f, g, h:[0, \infty] \rightarrow[0, \infty]$ are increasing superadditive function $\mathrm{S}^{1}$ vanishing at 0 , such that $h(x) \leqslant x$ for all $x$. In order to prove a) and b) one can use the inequality $a^{n}+b^{n} \leqslant(a+b)^{n}$.

Corollary 4.9. If $\mu$ is a subadditive monotone measure, or $f, g \in \mathcal{F}_{(X,[0, \infty])}$ are comonotone functions, then

$$
\mathrm{Su}_{+}^{n}(f+g) \leqslant \mathrm{Su}_{+}^{n}(f)+\mathrm{Su}_{+}^{n}(g)
$$

for all $n \geqslant 1$. Moreover, if (11) is valid for some $n$ and all $f, g \in \mathcal{F}_{(X,[0, \infty])}$, then $\mu$ is subadditive.

Proof. Put $Y=[0, \infty], \star=+$ and $a \nabla b=(a+b) \wedge \mu(X)$ or $\nabla=\vee$ in Theorem 4.7 and use Example 4.8 (d) with $q=1$.

Next, we give a partial solution to the problem posed in [30]. The question is how to compute the pseudo-decomposition integral of $n$-th order defined as

$$
\mathrm{I}_{n}^{\oplus, \odot}(\mu, f)=\sup \left\{\bigoplus_{i=1}^{n}\left(a_{i} \odot \mu\left(A_{i}\right)\right): \bigoplus_{i=1}^{n} a_{i} \mathbb{1}_{A_{i}} \leqslant f, a_{i} \in Y, A_{1} \subset \ldots \subset A_{n}\right\}
$$

based on a pseudo-addition $\oplus: Y^{2} \rightarrow Y$ and a $\oplus$-fitting pseudo-multiplication $\odot: Y \times[0, \mu(X)] \rightarrow Y$ (see [3, Definition 3.1 and 3.4]). The integral $\mathrm{I}_{n}^{\oplus, \odot}$ is also called the Benvenuti integral of $n$-th order. Our aim is to compute the integral $\mathrm{I}_{n}^{+, \wedge}(\mu, f)$. By the definition 12 we get

$$
\begin{aligned}
\mathrm{I}_{n}^{+, \wedge}(\mu, f) & =\sup \left\{\sum_{i=1}^{n}\left(a_{i} \wedge \mu\left(A_{i}\right)\right): \sum_{i=1}^{n} a_{i} \mathbb{1}_{A_{i}} \leqslant f, A_{1} \subset \ldots \subset A_{n}\right\} \\
& =\sup \left\{\sum_{i=1}^{n}\left(a_{i} \wedge \mu\left(A_{i}\right)\right): \sum_{i=1}^{n}\left(\sum_{k=i}^{n} a_{k}\right) \mathbb{1}_{A_{i} \backslash A_{i-1}} \leqslant f, A_{1} \subset \ldots \subset A_{n}\right\} \\
& =\sup \left\{\sum_{i=1}^{n}\left(\left(b_{i}-b_{i+1}\right) \wedge \mu\left(\left\{f \geqslant b_{i}\right\}\right)\right): 0=b_{n+1} \leqslant b_{n} \leqslant \ldots \leqslant b_{1} \leqslant \bar{y}\right\}
\end{aligned}
$$

with $b_{i}=\sum_{k=i}^{n} a_{k}$ and $A_{0}:=\emptyset$, but computation of the integral from formula (13) is still a difficult task. However, there is a connection with the lower $n$-Sugeno integral.

Theorem 4.10. For all $(\mu, f) \in \mathcal{M}_{(X, \mathcal{A})} \times \mathcal{F}_{(X, Y)}$ and $n \geqslant 1$

$$
\mathrm{I}_{n}^{+, \wedge}(\mu, f)=\mathrm{Su}_{+}^{n}(\mu, f) .
$$

\footnotetext{
${ }^{1}$ A function $f$ is superadditive if $f(a+b) \geqslant f(a)+f(b)$ for any $a, b$.
} 
Proof. See Appendix.

Combining the definition of $\mathrm{Su}_{+}^{n}(\mu, f)$ with Theorem 4.10 gives the following simple recurrence scheme

$$
\mathrm{I}_{n}^{+, \wedge}(\mu, f)=\sup _{y \in Y}\left\{y \wedge\left(\mu(\{f \geqslant y\})+\mathrm{I}_{n-1}^{+, \wedge}(\mu, f)\right)\right\}
$$

with $\mathrm{I}_{1}^{+, \wedge}(\mu, f):=\mathrm{Su}(f)$.

Example 4.11. Let $X=[0,1], f(x)=x$ and $\mu(A)=(\lambda(A))^{1 / 2}$, where $\lambda$ is the Lebesgue measure. Then

$$
\mathrm{I}_{n}=\frac{2 \mathrm{I}_{n-1}-1+\sqrt{5-4 \mathrm{I}_{n-1}}}{2}, \quad n=1,2, \ldots,
$$

where $\mathrm{I}_{n}:=\mathrm{I}_{n}^{+, \wedge}(\mu, f)$ and $\mathrm{I}_{0}:=0$. If $\mu(A)=(\lambda(A))^{2}$, then

$$
\mathrm{I}_{n}=\frac{3-\sqrt{5-4 \mathrm{I}_{n-1}}}{2}, \quad n=1,2, \ldots
$$

The next result provides a connection between the lower $n$-Sugeno integral and the generalized Choquet integral introduced in [9] and deeply studied in [29].

Theorem 4.12. Let $(\mu, f) \in \mathcal{M}_{(X, \mathcal{A})} \times \mathcal{F}_{(X, Y)}$ and $n \geqslant 2$. The lower $n$-Sugeno integral can be represented as

$$
\operatorname{Su}_{+}^{n}(f)=\inf \left\{\sum_{i=1}^{n}\left(\left(b_{i}-b_{i+1}\right) \vee \mu\left(\left\{f>b_{i}\right\}\right)\right): 0=b_{n+1} \leqslant b_{n} \leqslant \ldots \leqslant b_{1} \leqslant \bar{y}\right\} .
$$

Proof. See Appendix.

The lower 2-Sugeno integral is neither maxitive nor minitive homogeneous functional.

Example 4.13. Consider $f$ as in the Example 3.11. Let $\mu(B)=0.25$ and $\mu(X)=1$. Then $\operatorname{Su}_{+}^{2}(f)=$ 0.5 and $\mathrm{Su}_{+}^{2}\left((1 / 3) \mathbb{1}_{X} \vee f\right)=7 / 12$, so $\mathrm{Su}_{+}^{2}\left((1 / 3) \mathbb{1}_{X} \vee f\right)>(1 / 3) \vee \mathrm{Su}_{+}^{2}(f)$.

Example 4.14. Let $f=0.5 \mathbb{1}_{A}, \mu(A)=0.5, \mu(X)=1$ and $\star=\cdot$. After simple calculations, we get $\mathrm{Su}_{.}^{2}(f)=0.25$ and $\mathrm{Su}_{.}^{2}\left(0.1 \mathbb{1}_{X} \wedge f\right)=0.05$. Thus, $\operatorname{Su}^{2}\left(0.1 \mathbb{1}_{X} \wedge f\right)<0.1 \wedge \mathrm{Su}^{2}(f)$.

Now we give a sufficient condition for minitive/maxitive homogeneity.

Proposition 4.15. Suppose that $n \geqslant 2$ and $(\mu, f) \in \mathcal{M}_{(X, \mathcal{A})} \times \mathcal{F}_{(X, Y)}$.

(a) $\operatorname{Su}_{\star}^{n}\left(a \mathbb{1}_{X} \wedge f\right)=a \wedge \operatorname{Su}_{\star}^{n}(f)$ for any $a \in Y$ if $a \wedge(b \star(a \wedge c))=a \wedge(b \star c)$ for all $a, c \in Y$ and $b \in \mu(\mathcal{A})$.

(b) $\operatorname{Su}_{\star}^{n}\left(a \mathbb{1}_{X} \vee f\right)=a \vee \operatorname{Su}_{\star}^{n}(f)$ for any $a \in Y$ if $\mu(X) \geqslant \bar{y}$ and $b \wedge c \leqslant b \star c \leqslant b \vee c$ for all $b \in[0, \infty]$ and $c \in Y$. 
Proof. The proof of part (a) is similar to that of Proposition 3.12(a) (applying Theorem 4.5), so we omit it.

Now we show $(b)$ by induction. The proof for $n=2$ will be omitted as it is quite similar to the proof of the second induction step. From Theorem 4.5 and the induction hypothesis, we have

$$
\begin{aligned}
& \mathrm{Su}_{\star}^{n}\left(a \mathbb{1}_{X} \vee f\right)= \sup _{A}\left\{\left(a \vee \inf _{x \in A} f(x)\right) \wedge\left[\mu(A) \star\left(a \vee \mathrm{Su}_{\star}^{n-1}(f)\right)\right]\right\} \\
&=\sup _{A}\left\{\left(a \vee \inf _{x \in A} f(x)\right) \wedge\left[(\mu(A) \star a) \vee\left(\mu(A) \star \mathrm{Su}_{\star}^{n-1}(f)\right)\right]\right\} \\
&=\sup _{A}\left\{[a \wedge(\mu(A) \star a)] \vee\left[a \wedge\left(\mu(A) \star \mathrm{Su}_{\star}^{n-1}(f)\right)\right]\right. \\
&\left.\quad \vee\left[\inf _{x \in A} f(x) \wedge(\mu(A) \star a)\right] \vee\left[\inf _{x \in A} f(x) \wedge\left(\mu(A) \star \mathrm{Su}_{\star}^{n-1}(f)\right)\right]\right\},
\end{aligned}
$$

where we write $\sup _{A}$ instead of $\sup _{A \in \mathcal{A}}$. Furthermore

$$
\begin{gathered}
\operatorname{Su}_{\star}^{n}\left(a \mathbb{1}_{X} \vee f\right)=[a \wedge(\mu(X) \star a)] \vee\left[a \wedge\left(\mu(X) \star \operatorname{Su}_{\star}^{n-1}(f)\right)\right] \vee \sup _{A}\left\{\inf _{x \in A} f(x) \wedge(\mu(A) \star a)\right\} \\
\vee \sup _{A}\left\{\inf _{x \in A} f(x) \wedge\left(\mu(A) \star \mathrm{Su}_{\star}^{n-1}(f)\right)\right\} .
\end{gathered}
$$

By the assumption that $\mu(X) \star a \geqslant \mu(X) \wedge a=a$ and the fact that $a \wedge\left(\mu(X) \star \operatorname{Su}_{\star}^{n-1}(f)\right) \leqslant a$, we have

$$
\mathrm{Su}_{\star}^{n}\left(a \mathbb{1}_{X} \vee f\right)=a \vee \sup _{A}\left\{\inf _{x \in A} f(x) \wedge(\mu(A) \star a)\right\} \vee \mathrm{Su}_{\star}^{n}(f)
$$

Observe that

$$
\begin{aligned}
\sup _{A}\left\{\inf _{x \in A} f(x) \wedge(\mu(A) \star a)\right\} & \leqslant \sup _{A}\left\{\inf _{x \in A} f(x) \wedge(\mu(A) \vee a)\right\} \\
& =\sup _{A}\left\{\left(\inf _{x \in A} f(x) \wedge \mu(A)\right) \vee\left(\inf _{x \in A} f(x) \wedge a\right)\right\} \\
& \leqslant \operatorname{Su}(f) \vee a \leqslant \operatorname{Su}_{\star}^{n}(f) \vee a,
\end{aligned}
$$

where the last inequality follows from Proposition 4.4. By (15), we obtain $\operatorname{Su}_{\star}^{n}\left(a \mathbb{1}_{X} \vee f\right)=a \vee \operatorname{Su}_{\star}^{n}(f)$, as desired.

The condition in $(a)$ is satisfied if $x \star a \geqslant a$ for any $x$ and $a$, e.g. $x \star a:=\left(x^{p}+a^{p}\right)^{1 / p}$ for $p>0$. On the other hand, any OWA operator of the form $x \star y=p(x \wedge y)+(1-p)(x \vee y)$ satisfies the condition in $(b)$ for $p \in[0,1]$.

\section{Applications}

\section{(A) Scientometric indices}

We put $X=\mathbb{N}$, where $\mathbb{N}=\{1,2, \ldots\}$ denotes the set of all positive integers, and $\mu: 2^{\mathbb{N}} \rightarrow[0, \infty]$ is the counting measure, i.e., $\mu(A)=\operatorname{Card}(A)$ for any $A \in 2^{\mathbb{N}}$. A scholar with some publications is 
formally described by an infinite vector $\mathbf{x}=\left(x_{1}, x_{2}, \ldots\right)$, called a scientific record, where $x_{i} \in \mathbb{N}_{0}$ with $\mathbb{N}_{0}=\mathbb{N} \cup\{0\}$ such that $x_{1} \geqslant x_{2} \geqslant \ldots$. The positive value of $x_{i}$ gives the number of citations of $i$-th scholar publication, and the value $x_{i}=0$ means either a paper with zero citations or a nonexisting paper. From now on we consider the scientific records with $x_{1} \geqslant 1$. The $h$-index of $\mathbf{x}$ is defined as follows [23, 28]

$$
\mathrm{H}(\mathbf{x})=\max \left\{k: x_{k} \geqslant k\right\}=\max _{k}\left\{k \wedge x_{k}\right\}
$$

Note that there are several papers characterizing the Hirsch index via various axioms, e.g. [8, 32, 33, 42, An interesting axiom $\mathbf{H}(\mathbf{x})=\mathbf{H}\left(\mathbf{y}_{\mathbf{x}}\right)$ is called the symmetry of the $h$-index, see [43, Proposition 3.1]. Here $\mathbf{y}_{\mathbf{x}}:=\left(y_{1}, y_{2}, \ldots\right)$ is called the conjugate of $\mathbf{x}$ with $y_{i}=\sum_{k=1}^{\infty} \mathbb{1}_{\left\{x_{k} \geqslant i\right\}}$ providing the number of publications with at least $i$ citations.

As it is well known, the Hirsch index has some drawbacks. In order to compensate some defects of $h$-index, many authors have introduced new scientometric indices that lead to better discrimination of some types of data than $h$-index (see [28, 31, 42]). Here we discuss a few of them and show that the upper/lower $n$-Sugeno integrals generalize some known scientometric indices. Firstly, recall that Narukawa and Torra [39] have shown that $h$-index is the Sugeno integral with respect to counting measure. In consequence, the upper/lower $n$-Sugeno integral generalizes $h$-index too.

(i) Generalized Kosmulski index There are several modifications of $h$-index based on the input $k$, e.g. $h_{\lambda}(\mathbf{x})=\max \left\{k: x_{k} \geqslant \lambda k\right\}$ of Van Eck [40], $h(2)$-index $\mathrm{H} 2(\mathbf{x})=\max \left\{k: x_{k} \geqslant k^{2}\right\}$ of Kosmulski [27], or its extended version $\max \left\{k: x_{k} \geqslant k^{m}\right\}$ with $m=3,4, \ldots$ In general, for any nondecreasing function $s:[0, \infty] \rightarrow[0, \infty]$ the generalized Kosmulski index is given by $\mathrm{K}_{s}(\mathbf{x})=\max \left\{k: x_{k} \geqslant s(k)\right\}$, see $[13]^{2}$.

Now we will show the connection between generalized Kosmulski index and upper/lower 2-Sugeno integral. For this purpose consider $\circ_{s}:[0, \infty]^{2} \rightarrow[0, \infty]$ defined as $a \circ_{s} b=s(a)$ with $s:[0, \infty] \rightarrow[0, \infty]$ being a nondecreasing function such that $s(0)=0$. Immediately, $\circ_{s}$ is an admissible fusion as well as a link function with $Y=[0, \infty]$. Note that each scientific record $\mathbf{x}$ uniquely determines a function $f: X \rightarrow \mathbb{N}_{0}$ as $x_{i}=f(i)$, and vice versa. Hence, the notation $\operatorname{Su}_{2}^{\mathrm{o}_{s}}(\mathbf{x})$ is justified and

$$
\mathrm{Su}_{2}^{\mathrm{o}_{s}}(\mathbf{x})=\max _{k}\left\{s(k) \wedge \mu\left(\left\{i: x_{i} \geqslant k\right\}\right)\right\}=\max _{j}\left\{s\left(x_{j}\right) \wedge \mu\left(\left\{i: x_{i} \geqslant x_{j}\right\}\right)\right\} .
$$

Since $\mu$ is the counting measure, we get

$$
\mathrm{Su}_{2}^{\mathrm{o}_{s}}(\mathbf{x})=\max _{j}\left\{s\left(x_{j}\right) \wedge j\right\}=\max _{j}\left\{s\left(\mu\left(\left\{i: y_{i} \geqslant j\right\}\right)\right) \wedge j\right\}=\mathrm{Su}_{o_{s}}^{2}\left(\mathbf{y}_{\mathbf{x}}\right),
$$

\footnotetext{
${ }^{2}$ In order to get an integer-valued index, in the original paper authors consider the function $s: \mathbb{N}_{0} \rightarrow \mathbb{N}_{0}$ with $s(0)=0$ and $s(k) \geqslant 1$ for each $k \in \mathbb{N}$.
} 
where $\mathbf{y}_{\mathbf{x}}$ is the conjugate of $\mathbf{x}$. Moreover,

$$
\begin{aligned}
\mathrm{Su}_{\mathrm{o}_{s}}^{2}(\mathbf{x}) & =\max _{k}\left\{k \wedge s\left(\mu\left(\left\{i: x_{i} \geqslant k\right\}\right)\right)\right\}=\max _{k}\left\{x_{k} \wedge s\left(\mu\left(\left\{i: x_{i} \geqslant x_{k}\right\}\right)\right)\right\} \\
& =\max _{k}\left\{x_{k} \wedge s(k)\right\}=\max _{k}\left\{\mu\left(\left\{i: y_{i} \geqslant k\right\}\right) \wedge s(k)\right\}=\mathrm{Su}_{2}^{\mathrm{o}_{s}}\left(\mathbf{y}_{\mathbf{x}}\right) .
\end{aligned}
$$

It is easy to see that $\mathrm{Su}_{o_{s}}^{2}(\mathbf{x})$ and $\operatorname{Su}_{2}^{\mathrm{o}_{s}}(\mathbf{x})$ for $s(a)=a$ (under the convention $s(\infty)=\infty$ ) coincide with the $h$-index of $\mathbf{x}$. In consequence, this proves Proposition 31 from [43], i.e., the symmetry $\mathbf{H}(\mathbf{x})=\mathbf{H}\left(\mathbf{y}_{\mathbf{x}}\right)$ of $h$-index. However, the integrals $\mathrm{Su}_{2}^{\mathrm{o}_{s}}(\cdot)$ and $\mathrm{Su}_{\mathrm{o}_{s}}^{2}(\cdot)$ are not symmetric in general, i.e., the equalities $\mathrm{Su}_{2}^{\mathrm{o}_{s}}(\mathbf{x})=\mathrm{Su}_{2}^{\circ_{s}}\left(\mathbf{y}_{\mathbf{x}}\right)$ and $\mathrm{Su}_{\mathrm{o}_{s}}^{2}(\mathbf{x})=\operatorname{Su}_{\mathrm{o}_{s}}^{2}\left(\mathbf{y}_{\mathbf{x}}\right)$ need not hold for each $s$ and $\mathbf{x}$.

Example 5.1. For $s(a)=2 a$ and the scientific record $\mathbf{x}=(3,0, \ldots)$ with $\mathbf{y}_{\mathbf{x}}=(1,1,1,0, \ldots)$ we have $\mathrm{Su}_{2}^{\circ_{s}}(\mathbf{x})=1=\operatorname{Su}_{\circ_{s}}^{2}\left(\mathbf{y}_{\mathbf{x}}\right)$, but $\operatorname{Su}_{2}^{\circ_{s}}\left(\mathbf{y}_{\mathbf{x}}\right)=2=\operatorname{Su}_{o_{s}}^{2}(\mathbf{x})$. Note that for $s(a)=\lambda a$ with $\lambda>0$ it follows from (17) that $\mathrm{Su}_{\mathrm{o}_{s}}^{2}(\mathbf{x})=\max _{k}\left\{x_{k} \wedge(\lambda k)\right\}$. This index was introduced in [20, Definition 2].

Proposition 5.2. Let $\circ_{s}:[0, \infty]^{2} \rightarrow[0, \infty]$ be such that $a \circ_{s} b=s(a)$ with $s:[0, \infty] \rightarrow[0, \infty]$ being an increasing and continuous function such that $s(0)=0$. Then for each scientific record $\mathbf{x}$ we have

(a) $\mathrm{K}_{s}(\mathbf{x})=\mathrm{Su}_{2}^{\circ\lfloor\widehat{s}\rfloor}(\mathbf{x})$,

(b) $\mathrm{K}_{s}(\mathbf{x})=\left\lfloor\mathrm{Su}_{2}^{\mathrm{o}^{\widehat{s}}}(\mathbf{x})\right\rfloor=\left\lfloor\widehat{s}\left(\mathrm{Su}_{\mathrm{o}_{s}}^{2}(\mathbf{x})\right)\right\rfloor$,

where $\lfloor\cdot\rfloor$ is the floor function and $\widehat{s}=s^{-1}$.

Proof. Observe that $\mathrm{K}_{s}(\mathbf{x})=\max \left\{k: s^{-1}\left(x_{k}\right) \geqslant k\right\}=\max \left\{k:\left\lfloor s^{-1}\left(x_{k}\right)\right\rfloor \geqslant k\right\}=\max _{k}\left\{k \wedge\left\lfloor s^{-1}\left(x_{k}\right)\right\rfloor\right\}$. From (16), we get $\mathrm{K}_{s}(\mathbf{x})=\mathrm{Su}_{2}^{{ }^{\lfloor}{ }^{[\hat{s}\rfloor}}(\mathbf{x})$, as ${ }_{\lfloor\hat{s}\rfloor}$ is an admissible fusion function. Next, note that $\mathrm{Su}_{2}{ }^{\lfloor\hat{s}\rfloor}(\mathbf{x})=\left\lfloor\max _{k}\left\{k \wedge s^{-1}\left(x_{k}\right)\right\}\right\rfloor$, since $\lfloor k \wedge a\rfloor=k \wedge\lfloor a\rfloor$ for each $a \geqslant 0$ and $k \in \mathbb{N}$ and $\max _{k} g\left(z_{k}\right)=$ $g\left(\max _{k} z_{k}\right)$ for any nondecreasing function $g$. Using (16) again, we obtain $\mathrm{K}_{s}(\mathbf{x})=\left\lfloor\mathrm{Su}_{2}^{\circ}(\mathbf{x})\right\rfloor$. Moreover, $\left.\mathrm{K}_{s}(\mathbf{x})=\left\lfloor s^{-1}\left(\max _{k}\left\{s(k) \wedge x_{k}\right)\right\}\right)\right\rfloor=\left\lfloor\widehat{s}\left(\mathrm{Su}_{\circ_{s}}^{2}(\mathbf{x})\right)\right\rfloor$, where the latter equality follows from (17).

All the above considerations are true also for the upper/lower $n$-Sugeno integral for any $n \geqslant 2$.

(ii) Upper and lower 2 - $h$-indices We return back to the original indices our motivation comes from. Indeed, Mesiar and Gągolewski [28] introduced the upper 2- $h$-index and the lower 2- $h$-index of a scientific record $\mathbf{x}$ as follows:

$$
\begin{aligned}
& \mathrm{H}_{2}^{u}(\mathbf{x})=\max _{k}\left\{(k+\mathrm{H}(\mathbf{x})) \wedge x_{k}\right\}, \\
& \mathrm{H}_{2}^{l}(\mathbf{x})=\mathrm{H}(\mathbf{x})+\max _{k}\left\{(k-\mathrm{H}(\mathbf{x}))_{+} \wedge x_{k}\right\}=\max _{k}\left\{k \wedge\left(x_{k}+\mathrm{H}(\mathbf{x})\right)\right\},
\end{aligned}
$$

where $a_{+}=\max (a, 0)$. In other words, upper 2 - $h$-index is $h$-index increased by the value of $h$-index calculated for the scientist's output after removing $h$ citations from each work. On the other hand, lower 2$h$-index is $h$-index increased by the value of $h$-index of a scientific record $\left.\mathbf{x}\right|_{\mathrm{H}(\mathbf{x})}=\left(x_{\mathrm{H}(\mathbf{x})+1}, x_{\mathrm{H}(\mathbf{x})+2}, \ldots\right)$. The latter $h$-index of $\left.\mathbf{x}\right|_{\mathbf{H}(\mathbf{x})}$ corresponds to $h$-index of $\mathbf{x}$ without publications in the Hirsch core, cf. [34]. 
Example 5.3. Let $\mathbf{x}=(6,6,4,3,1,1,1,0, \ldots)$. Clearly, $\mathbf{H}(\mathbf{x})=3$, and $\left.\mathbf{x}\right|_{\mathbf{H}(\mathbf{x})}=(3,1,1,1,0, \ldots)$ is the scientific record obtained from $\mathbf{x}$ after removing the Hirsch core, i.e., the first three papers. Since $\mathrm{H}\left(\left.\mathbf{x}\right|_{\mathbf{H}(\mathbf{x})}\right)=1$, we have $\mathrm{H}_{2}^{l}(\mathbf{x})=4$. Analogously, $\mathbf{H}_{2}^{u}(\mathbf{x})=5$, see Fig. 1 .
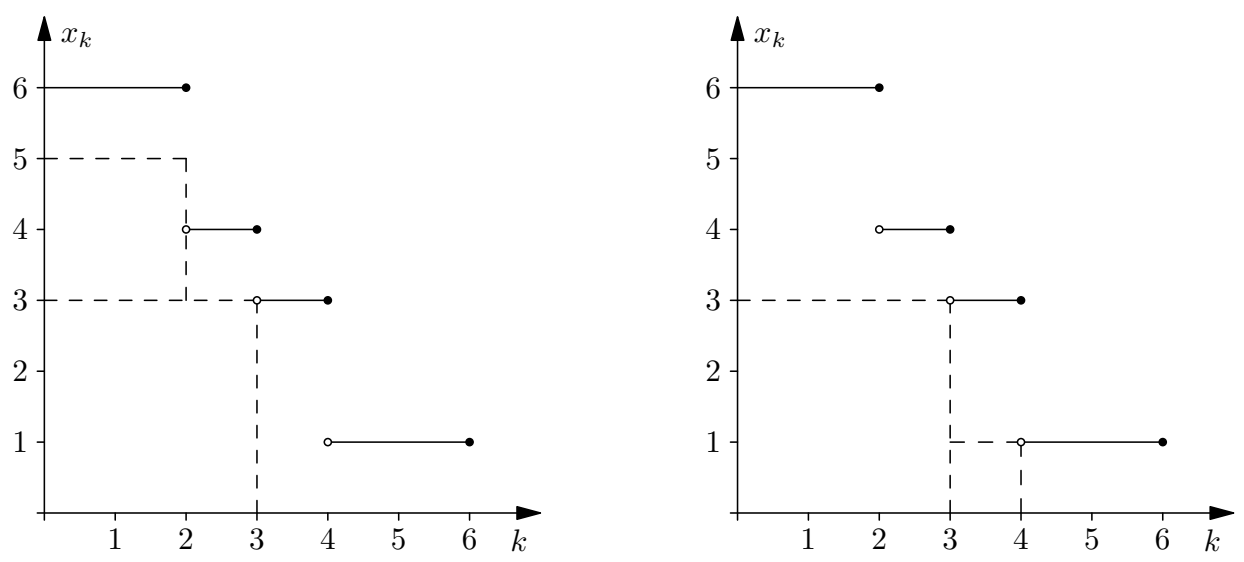

Figure 1: Illustration of formula 18$)$ for $\mathrm{H}_{2}^{u}(\mathbf{x})=5$ (left) and 19$)$ for $\mathrm{H}_{2}^{l}(\mathbf{x})=4$ (right).

Proposition 5.4. For each scientific record $\mathbf{x}$ we have $\mathrm{Su}_{2}^{+}(\mathbf{x})=\mathrm{H}_{2}^{l}(\mathbf{x})=\mathrm{Su}_{+}^{2}\left(\mathbf{y}_{\mathbf{x}}\right)$ and $\mathrm{Su}_{+}^{2}(\mathbf{x})=$ $\mathrm{H}_{2}^{u}(\mathbf{x})=\mathrm{Su}_{2}^{+}\left(\mathbf{y}_{\mathbf{x}}\right)$.

Proof. For proving the statements, the admissible fusion function and the link function is $\circ_{s}=+$. Repeating the considerations from 160 and 17 we finish the proof.

(iii) $\mathrm{H}_{\alpha}$ and $\mathrm{H}^{\beta}$-indices Next we show that the indices $\mathrm{H}_{\alpha}$ and $\mathrm{H}^{\beta}$ recently introduced in [24] as

$$
\begin{aligned}
& \mathrm{H}_{\alpha}(\mathbf{x})=\max _{k}\left\{\left\lfloor\left(x_{k} / \alpha\right) \wedge \mu\left(\left\{i: x_{i} \geqslant x_{k}\right\}\right)\right\rfloor\right\}=\max _{k}\left\{\left\lfloor\left(x_{k} / \alpha\right) \wedge k\right\rfloor\right\}, \quad \alpha>0, \\
& \mathrm{H}^{\beta}(\mathbf{x})=\left\lceil\max _{k}\left\{x_{k} \wedge\left(\mu\left(\left\{i: x_{i} \geqslant x_{k}\right\}\right) / \beta\right)\right\}\right\rceil=\left\lceil\max _{k}\left\{x_{k} \wedge(k / \beta)\right\}\right\rceil, \quad \beta>0,
\end{aligned}
$$

are also a special case of upper/lower Sugeno integral. Here, $\lceil\cdot\rceil$ is the ceiling of a real number. Index $\mathrm{H}_{\alpha}$ is able to compensate a lower number of citations and $\mathrm{H}^{\beta}$ compensates a lower number of papers.

Proposition 5.5. For each scientific record $\mathbf{x}$ we have

(i) $\mathrm{H}_{\alpha}(\mathbf{x})=\mathrm{Su}_{2}^{o_{s}}(\mathbf{x})$ with $s(a)=\lfloor a / \alpha\rfloor$,

(ii) $\mathrm{H}^{\beta}(\mathbf{x})=\mathrm{Su}_{\mathrm{o}_{s}}^{2}(\mathbf{x})$ with $s(a)=\lceil a / \beta\rceil$.

Proof. (i) Based on (16) we have $\mathrm{Su}_{2}^{\mathrm{o}_{s}}(\mathbf{x})=\max _{k}\left\{\left\lfloor x_{k} / \alpha\right\rfloor \wedge k\right\}$. To finish the proof one can use the fact that $\lfloor a \wedge k\rfloor=\lfloor a\rfloor \wedge k$ for each $a \geqslant 0$ and $k \in \mathbb{N}$.

(ii) Using (17) we get $\mathrm{Su}_{\circ_{s}}^{2}(\mathbf{x})=\max _{k}\left\{x_{k} \wedge\lceil k / \beta\rceil\right\}$. Since $\lceil a \wedge k\rceil=\lceil a\rceil \wedge k$ for $a \geqslant 0$ and $k \in \mathbb{N}$, so $\mathrm{Su}_{\mathrm{o}_{s}}^{2}(\mathbf{x})=\max _{k}\left\{\left\lceil x_{k} \wedge(k / \beta)\right\rceil\right\}$. To get the statement, use $\max _{k} g\left(z_{k}\right)=g\left(\max _{k} z_{k}\right)$ for any nondecreasing function $g$. 
Using the similar arguments as in the proof of Proposition 5.5(ii) one can show that $\mathrm{H}_{\alpha}(\mathbf{x})=$ $\left\lfloor\mathrm{Su}_{2}^{\mathrm{o}_{s}}(\mathbf{x})\right\rfloor$ with $s(a)=a / \alpha$ and $\mathbf{H}^{\beta}(\mathbf{x})=\left\lceil\mathrm{Su}_{o_{s}}^{2}(\mathbf{x})\right\rceil$ with $s(a)=a / \beta$. Thence and from Proposition 5.2 (b) we conclude that $\mathrm{H}_{\alpha}$ is a special case of the generalized Kosmulski index $\mathrm{K}_{s}$ with $s(k)=\alpha k$.

(iv) Iterated $h$-index In 2009 in García-Pérez [18, 19] considered a multidimensional $h$-index and showed that the additional components are useful to distinguish individuals with the same $h$-index. This approach has been studied further in [4] in order to provide its axiomatic characterization. Formally, the iterated $h$-index $\mathrm{iH}$ of a scientific record $\mathbf{x}$ is a vector $\mathrm{iH}(\mathbf{x})=\left(\mathrm{iH}_{1}(\mathbf{x}), \mathrm{iH}_{2}(\mathbf{x}), \ldots\right)$ with the components $\mathrm{iH}_{n}(\mathbf{x})$ defined for each $n \in \mathbb{N}$ by

$$
\mathrm{iH}_{n}(\mathbf{x})=\max _{k}\left\{k \wedge x_{\mathrm{iH}_{0}(\mathbf{x})+\ldots+\mathrm{iH}_{n-1}(\mathbf{x})+k}\right\}
$$

with $\mathrm{iH}_{0}(\mathbf{x}):=0$. Clearly, $\mathrm{iH}_{1}(\mathbf{x})=\mathrm{H}(\mathbf{x})$ and $\mathrm{iH}_{1}(\mathbf{x}) \geqslant \mathrm{iH}_{2}(\mathbf{x}) \geqslant \ldots$ Also, it is easy to see that $\mathrm{iH}_{n}(\mathbf{x})=\mathrm{Su}_{+}^{n}(\mathbf{x})-\mathrm{Su}_{+}^{n-1}(\mathbf{x})$. Thus,

Proposition 5.6. For each scientific record $\mathbf{x}$ and each $n \in \mathbb{N}$ we have $\mathrm{Su}_{+}^{n}(\mathbf{x})=\sum_{k=1}^{n} \mathrm{iH}_{k}(\mathbf{x})$.

(v) $p$-index and $c$-index It follows from Proposition 5.6 that the functional defined by $\mathrm{Su}_{+}^{\infty}(\mathbf{x}):=$ $\sup _{n} \mathrm{Su}_{+}^{n}(\mathbf{x})$ gives a number of publications with at least one citation. This index is known as the $p$ index (see [40, Definition 2.5]). On the other hand, the number $\operatorname{Su}_{\infty}^{+}(\mathbf{x}):=\sup _{n} \operatorname{Su}_{n}^{+}(\mathbf{x})=x_{1}$ represents a number of citations of the most important paper and it is called the c-index [40, Definition 2.6], or the maximum-index [42, Definition 2.5]. The $p$ - and $c$-indices measure almost completely opposite aspects of the performance of a researcher. The $p$-index can be seen as a measure of productivity with focusing on productivity (i.e., number of papers) and paying almost no attention to impact (i.e., number of times a paper has been cited). On the other hand, the $c$-index can be seen as a measure of impact with focusing on impact and paying no attention at all to productivity. For instance, it prefers a single highly cited paper over a large number of slightly lower cited papers. Finally, the $s$-index defined by $\mathrm{s}(\mathbf{x})=\sum_{i=1}^{\infty} x_{i}$ equals the total number of citations of all papers published by the scientist. Thus, the $s$-index takes into account all papers published by a scientist and not only the most cited paper (as in $c$-index).

\section{(B) Aggregation functions}

Nowadays, aggregation processes naturally appear in almost every discipline and importance of aggregation functions may be seen in various applications including data fusion, decision making, computer science, social choice, etc. We shall show here that both the upper $n$-Sugeno and the lower $n$-Sugeno 
integrals are new aggregation functions under some mild assumptions on maps $\circ$ and $\star$ with a very natural max-min-type representations. Firstly we recall the definition of an aggregation function.

Definition 5.7. [2, Definition 1.5] A function A: $[0, \bar{y}]^{m} \rightarrow[0, \bar{y}]$ is said to be an m-ary aggregation function, if it is nondecreasing and it satisfies the boundary conditions $\mathrm{A}(0, \ldots, 0)=0$ and $\mathrm{A}(\bar{y}, \ldots, \bar{y})=$ $\bar{y}$.

Put $X=\{1,2, \ldots, m\}, Y=[0, \bar{y}]$ and $\mu \in \mathcal{M}_{(X, \mathcal{A})}$ such that $\mu(X) \geqslant \bar{y}$. For $n \geqslant 2$ the upper $n$-Sugeno integral $\mathrm{Su}_{n}^{\circ}(\mathbf{x})$ with $\mathbf{x}=\left(x_{1}, \ldots, x_{m}\right), x_{i} \in Y$, and an admissible fusion map $\circ$ satisfying $\bar{y} \circ \bar{y}=\bar{y}$, is an $m$-ary aggregation function. Indeed, from Proposition 3.3 (d) we get $\operatorname{Su}_{n}^{\circ}\left(\bar{y} \mathbb{1}_{X}\right)=\bar{y}$ and $\mathrm{Su}_{n}^{\circ}\left(0 \mathbb{1}_{X}\right)=0$ for all $n$. Monotonicity follows from Proposition 3.2 (a). Additionally, the lower $n$ Sugeno integral $\mathrm{Su}_{\star}^{n}(\mathbf{x})$ for $n \geqslant 2$ is also an aggregation function if the link map $\star:[0, \infty] \times Y \rightarrow[0, \infty]$ is such that $\star \geqslant \wedge$. In fact, the monotonicity follows from Proposition 4.2 (a), and by Proposition 4.3 (f) we have $\operatorname{Su}_{\star}^{n}\left(\bar{y} \mathbb{1}_{X}\right)=\bar{y}$ and $\operatorname{Su}_{\star}^{n}\left(0 \mathbb{1}_{X}\right)=0$. Moreover, for each $n \in \mathbb{N}$ we obtain

$$
\mathrm{Su}_{n+1}^{\circ}(\mathbf{x})=\bigvee_{T \subset X}\left[\left(\left(\bigwedge_{i \in T} x_{i}\right) \circ \mathrm{Su}_{n}^{\circ}(\mathbf{x})\right) \wedge \mu(T)\right], \quad \mathrm{Su}_{\star}^{n+1}(\mathbf{x})=\bigvee_{T \subset X}\left[\left(\bigwedge_{i \in T} x_{i}\right) \wedge\left(\mu(T) \star \mathrm{Su}_{\star}^{n}(\mathbf{x})\right)\right]
$$

(see Theorem 3.6 and Theorem 4.5 for $\mathcal{A}=2^{X}$ ) providing the weighted max-min-type representations of the two sequences of aggregation functions.

\section{Conclusions}

Generalizing the upper and lower 2- $h$-indices of Mesiar and Gągolewski [28] we have constructed upper and lower $n$-Sugeno integrals via iterating the Sugeno integral. These two classes of new functionals also include the generalized Kosmulski index [13] and $\mathrm{H}_{\alpha}$-index [24]. We have examined some of their universal mathematical properties that are useful in various fields such as scientometry, theory of integral and aggregation functions. Since there is only a few number of papers combining the above fields, the present paper stimulates a deeper study of the relationship between nonlinear functionals and scientometric indices. Thus, an applied research is supported by a theoretical research.

As a by-product, we have partially solved the question on computation of certain pseudo-decomposition integral providing the representation of Benvenuti integral of $n$-th order with respect to operations $\oplus=+$ and $\odot=\wedge$ as the lower $n$-Sugeno integral with respect to + . So, our approach provides a new way to look at pseudo-decomposition integrals and possibilities of their computation. 


\section{Acknowledgement}

Authors would like to express their sincere thanks and gratitude to anonymous reviewers for their thoughtful suggestions toward the improvement of the paper. This work was supported by the Slovak Research and Development Agency under the contract No. APVV-16-0337. The work is also cofinanced by bilateral call Slovak-Poland grant scheme No. SK-PL-18-0032 together with the Polish National Agency for Academic Exchange under the contract No. PPN/BIL/2018/1/00049/U/00001.

\section{Appendix}

Proof of Theorems 3.5 and 4.6. The arguments are similar to those of [6, Theorem 2]. Put

$$
S_{n+1}=\sup _{t \in Y}\left\{\left(t \circ S_{n}\right) \wedge\left(\mu(\{f \geqslant t\}) \star S_{n}\right)\right\}, \quad Z_{n+1}=\inf _{t \in Y}\left\{\left(t \circ Z_{n}\right) \vee\left(\mu(\{f>t\}) \star Z_{n}\right)\right\}
$$

for all $n \geqslant 1$ with $S_{1}=\operatorname{Su}(f)=Z_{1}$, where

(A) the map $\circ$ is given in Theorem 3.5 and $a \star b=a$, or

(B) the map $\star$ is defined in Theorem 4.6 and $a \circ b=a$.

Assume that $S_{1}>0$ as if $S_{1}=0$, then $S_{n}=0=Z_{n}$ for all $n$ (see Propositions 3.3 (a) and 4.3 (a)). By induction we show that $S_{n}=Z_{n}$ for all $n$. Clearly $S_{1}=Z_{1}$. Suppose that $S_{k}=Z_{k}$ for all $k \leqslant n$. Set

$$
I:=\left\{t \in Y: \mu(\{f \geqslant t\}) \star S_{n} \geqslant t \circ S_{n}\right\}, \quad J:=\left\{t \in Y: \mu(\{f>t\}) \star S_{n} \geqslant t \circ S_{n}\right\} .
$$

Clearly, $J \subset I$ and $0 \in J$ in the case (B). In the case (A) we have $a \star b=a$, so we find that

$$
\begin{aligned}
0 \circ S_{n} & \leqslant S_{n}=\inf _{t \in Y}\left\{\left(t \circ S_{n-1}\right) \vee \mu(\{f>t\})\right\} \\
& \leqslant\left(0 \circ S_{n-1}\right) \vee \mu(\{f>0\}) \leqslant S_{n-1} \vee \mu(\{f>0\}) \\
& \leqslant \ldots \leqslant S_{1} \vee \mu(\{f>0\})=\mu(\{f>0\}),
\end{aligned}
$$

as $0 \circ y \leqslant y$ and $S_{1}=\operatorname{Su}(f)=\inf _{t \in Y}\{t \vee \mu(\{f>t\})\} \leqslant \mu(\{f>0\})$. In consequence, $0 \in J$ in both cases. Since $t \mapsto t \circ S$ is nondecreasing and $t \mapsto \mu(\{f>t\}) \star S_{n}$ is nonincreasing, we have $I=[0, a]$ or $I=[0, a)$ and $J=[0, b]$ or $J=[0, b)$ with $b \leqslant a$. We need to show that $a=b$. Suppose that $b<a$. Hence by the definition of $I$ and $J$, we have

$$
\mu(\{f>t\}) \star S_{n}<t \circ S_{n} \leqslant \mu(\{f \geqslant t\}) \star S_{n}
$$

for any $t \in(b, a)$. Let $b<d<c<a$. As $\{f \geqslant c\} \subset\{f>d\}$, we get

$$
\mu(\{f \geqslant c\}) \star S_{n} \leqslant \mu(\{f>d\}) \star S_{n}<d \circ S_{n} \leqslant c \circ S_{n} \leqslant \mu(\{f \geqslant c\}) \star S_{n},
$$


a contradiction.

For each interval $D$, let $D^{c}=[0, \bar{y}] \backslash D$. By continuity of the maps $t \mapsto t \circ s$ and $t \mapsto t \star s$ we obtain

$$
\begin{aligned}
& S_{n+1}=\sup _{t \in Y}\left\{\left(t \circ S_{n}\right) \wedge\left(\mu(\{f \geqslant t\}) \star S_{n}\right)\right\}=\sup _{t \in I}\left\{t \circ S_{n}\right\} \vee \sup _{t \in I^{c}}\left\{\mu(\{f \geqslant t\}) \star S_{n}\right\} \\
& = \begin{cases}\bar{y} \circ S_{n} & \text { if } I=[0, \bar{y}], \\
\left(a \circ S_{n}\right) \vee\left(\mu\left(\left\{f \geqslant a^{+}\right\}\right) \star S_{n}\right) & \text { if } I=[0, a], a<\bar{y}, \\
\left(a \circ S_{n}\right) \vee\left(\mu(\{f \geqslant a\}) \star S_{n}\right) & \text { if } I=[0, a), a \leqslant \bar{y}\end{cases}
\end{aligned}
$$

with the convention that $\sup _{\emptyset}=0$ and $\inf _{\emptyset}=\infty$. Observe that

- if $I=[0, a]$ for $a<\bar{y}$, then from the definition of $I, \mu\left(\left\{f \geqslant a^{+}\right\}\right) \star S_{n} \leqslant a \circ S_{n}$, so $S_{n+1}=a \circ S_{n}$,

- if $I=[0, a)$ for $a \leqslant \bar{y}$, we have $\mu(\{f \geqslant a\}) \star S_{n}<a \circ S_{n}$, so $S_{n+1}=a \circ S_{n}$.

This implies that $S_{n+1}=a \circ S_{n}$. Further, we have

$$
\begin{aligned}
& Z_{n+1}=\inf _{t \in Y}\left\{\left(t \circ S_{n}\right) \vee\left(\mu(\{f>t\}) \star S_{n}\right)\right\}=\inf _{t \in J}\left\{\mu(\{f>t\}) \star S_{n}\right\} \wedge \inf _{t \in J^{c}}\left\{t \circ S_{n}\right\} \\
& = \begin{cases}0 \star S_{n} & \text { if } J=[0, \bar{y}], \\
\left(a \circ S_{n}\right) \wedge\left(\mu(\{f>a\}) \star S_{n}\right) & \text { if } J=[0, a], a<\bar{y}, \\
\left(a \circ S_{n}\right) \wedge\left(\mu\left(\left\{f>a^{-}\right\}\right) \star S_{n}\right) & \text { if } J=[0, a), a \leqslant \bar{y}\end{cases} \\
& = \begin{cases}0 \star S_{n} & \text { if } J=[0, \bar{y}], \\
a \circ S_{n} & \text { if } J=[0, a], a<\bar{y} \text { or } J=[0, a), a \leqslant \bar{y},\end{cases}
\end{aligned}
$$

as

- if $J=[0, a]$ and $a<\bar{y}$, then $\mu(\{f>a\}) \star S_{n} \geqslant a \circ S_{n}$, so $Z_{n+1}=a \circ S_{n}$,

- if $J=[0, a)$ and $a \leqslant \bar{y}$, then $\mu\left(\left\{f>a^{-}\right\}\right) \star S_{n} \geqslant a \circ S_{n}$, and so $Z_{n+1}=a \circ S_{n}$.

Consequently, we need to show that $S_{n+1}=Z_{n+1}$ if $J=[0, \bar{y}]=I$. Indeed, we have $S_{n+1}=\bar{y} \circ S_{n}$ and $Z_{n+1}=0 \star S_{n}$. Moreover,

$$
0 \star S_{n}=\mu(\{f>\bar{y}\}) \star S_{n} \geqslant \bar{y} \circ S_{n} .
$$

In the case (A), we have $0=0 \star S_{n} \geqslant \bar{y} \circ S_{n} \geqslant 0$, so $S_{n+1}=Z_{n+1}$. In the case (B), $S_{n+1}=\bar{y}$, $Z_{n+1}=0 \star S_{n}$ and $0 \star S_{n} \geqslant \bar{y}$. As $S_{n} \geqslant 0 \star S_{n}$ and $S_{n} \leqslant \bar{y}$, we get $0 \star S_{n}=\bar{y}$, and so $S_{n+1}=Z_{n+1}$. The proof is complete.

Proof of Theorem 4.10. We begin with the formula (13). It is clear that $\mathrm{I}_{1}^{+, \wedge}(\mu, f)=\operatorname{Su}(f)=$ $\mathrm{Su}_{+}^{1}(f)$. From Lemma 2.1 (e) it follows that if $\mathrm{Su}(f)=0$, then $\mu(\{f \geqslant t\})=0$ for all $t>0$. Hence by Proposition $4.3(\mathrm{a}), \mathrm{I}_{n}^{+, \wedge}(\mu, f)=0=\mathrm{Su}_{+}^{n}(f)$ and the assertion holds for any $n$. 
From now on let us assume $\mathrm{Su}(f)>0$. To get a better understanding, we first consider the case $n=2$, that is, we show that $\operatorname{Su}_{+}^{2}(f)=\sup _{b_{2} \in Y} M_{2}\left(b_{2}\right)$, where

$$
M_{2}\left(b_{2}\right)=\left(b_{2} \wedge \mu\left(\left\{f \geqslant b_{2}\right\}\right)\right)+\sup _{b_{1} \geqslant b_{2}}\left\{\left(b_{1}-b_{2}\right) \wedge \mu\left(\left\{f \geqslant b_{1}\right\}\right)\right\} .
$$

Clearly, $b_{2} \wedge \mu\left(\left\{f \geqslant b_{2}\right\}\right) \leqslant \operatorname{Su}(f)$, so $b_{2} \wedge \mu\left(\left\{f \geqslant b_{2}\right\}\right) \leqslant b_{2} \wedge \operatorname{Su}(f)$ and

$$
\begin{aligned}
M_{2}\left(b_{2}\right) & \leqslant\left(b_{2} \wedge \operatorname{Su}(f)\right)+\sup _{b_{1} \geqslant b_{2} \wedge \operatorname{Su}(f)}\left\{\left(b_{1}-\left(b_{2} \wedge \operatorname{Su}(f)\right)\right) \wedge \mu\left(\left\{f \geqslant b_{1}\right\}\right)\right\} \\
& =\sup _{b_{1} \geqslant b_{2} \wedge \operatorname{Su}(f)}\left\{b_{1} \wedge\left(\mu\left(\left\{f \geqslant b_{1}\right\}\right)+\left(b_{2} \wedge \operatorname{Su}(f)\right)\right)\right\} \\
& \leqslant \sup _{b_{1} \geqslant b_{2} \wedge \operatorname{Su}(f)}\left\{b_{1} \wedge\left(\mu\left(\left\{f \geqslant b_{1}\right\}\right)+\operatorname{Su}(f)\right)\right\} \\
& =\operatorname{Su}(f)+\sup _{b_{1} \geqslant b_{2} \wedge \operatorname{Su}(f)}\left\{\left(b_{1}-\operatorname{Su}(f)\right) \wedge \mu\left(\left\{f \geqslant b_{1}\right\}\right)\right\} .
\end{aligned}
$$

Since $\sup _{b_{1} \in\left[b_{2} \wedge \operatorname{Su}(f), \operatorname{Su}(f)\right]}\left\{\left(b_{1}-\mathrm{Su}(f)\right) \wedge \mu\left(\left\{f \geqslant b_{1}\right\}\right)\right\}=0$, we have

$$
M_{2}\left(b_{2}\right) \leqslant \sup _{b_{1} \geqslant \operatorname{Su}(f)}\left\{b_{1} \wedge\left(\mu\left(\left\{f \geqslant b_{1}\right\}\right)+\operatorname{Su}(f)\right)\right\}=\operatorname{Su}_{+}^{2}(f) .
$$

From the above it follows that

$$
\sup _{b_{2} \in Y} M_{2}\left(b_{2}\right) \leqslant \operatorname{Su}_{+}^{2}(f) .
$$

Now we show that the reverse inequality holds in 20 . Recall that $\operatorname{Su}(f)>0$. Evidently

$$
\sup _{b_{2} \in Y} M_{2}\left(b_{2}\right) \geqslant \lim _{b_{2}} \operatorname{Sin}_{\operatorname{Su}(f)} M_{2}\left(b_{2}\right) .
$$

By Lemma 2.1(c), we get

$$
\begin{aligned}
\lim _{b_{2} / \mathrm{Su}(f)} M_{2}\left(b_{2}\right) & =\mathrm{Su}(f)+\lim _{b_{2} / \operatorname{Su}_{(f)}} \sup _{b_{1} \geqslant b_{2}}\left\{\left(b_{1}-b_{2}\right) \wedge \mu\left(\left\{f \geqslant b_{1}\right\}\right)\right\} \\
& \geqslant \mathrm{Su}(f)+\lim _{b_{2} / \operatorname{Su}_{(f)}} \sup _{b_{1} \geqslant b_{2}}\left\{\left(b_{1}-\mathrm{Su}(f)\right) \wedge \mu\left(\left\{f \geqslant b_{1}\right\}\right)\right\} \\
& =\mathrm{Su}(f)+\sup _{b_{1} \geqslant \operatorname{Su}(f)}\left\{\left(b_{1}-\mathrm{Su}(f)\right) \wedge \mu\left(\left\{f \geqslant b_{1}\right\}\right)\right\} .
\end{aligned}
$$

Thus, $\sup _{a_{2} \in Y} M_{2}\left(a_{2}\right) \geqslant \mathrm{Su}_{+}^{2}(f)$, so there is the equality in 20$)$, as claimed.

Now, we show that the assertion (14) holds for all $n>2$. Observe that

$$
\mathrm{I}_{n}^{+, \wedge}(\mu, f)=\sup _{b_{n} \in Y} M_{n}\left(b_{n}, b_{n+1}\right),
$$

where $b_{n+1}=0$ and $M_{n}$ is defined recursively using the formula

$$
M_{k}\left(b_{k}, b_{k+1}\right):=\left[\left(b_{k}-b_{k+1}\right) \wedge \mu\left(\left\{f \geqslant b_{k}\right\}\right)\right]+\sup _{b_{k-1} \geqslant b_{k}} M_{k-1}\left(b_{k-1}, b_{k}\right)
$$


for $k=2, \ldots, n$ with the initial condition $M_{1}\left(b_{1}, b_{2}\right)=\left(b_{1}-b_{2}\right) \wedge \mu\left(\left\{f \geqslant b_{1}\right\}\right)$. Put $M_{k}^{*}\left(b_{k}\right):=$ $\sup _{b_{k-1} \geqslant b_{k}} M_{k-1}\left(b_{k-1}, b_{k}\right)$ for $k \geqslant 2$. Mimicking the proof for $n=2$, we obtain

$$
\begin{aligned}
M_{n}\left(b_{n}, b_{n+1}\right) \leqslant & \left(b_{n} \wedge \operatorname{Su}(f)\right)+\sup _{b_{n-1} \geqslant b_{n}}\left\{\left[\left(b_{n-1}-b_{n}\right) \wedge \mu\left(\left\{f \geqslant b_{n-1}\right\}\right)\right]+M_{n-1}^{*}\left(b_{n-1}\right)\right\} \\
\leqslant & \left(b_{n} \wedge \operatorname{Su}(f)\right) \\
& +\sup _{b_{n-1} \geqslant b_{n} \wedge \operatorname{Su}(f)}\left\{\left[\left(b_{n-1}-\left(b_{n} \wedge \operatorname{Su}(f)\right)\right) \wedge \mu\left(\left\{f \geqslant b_{n-1}\right\}\right)\right]+M_{n-1}^{*}\left(b_{n-1}\right)\right\} \\
= & \sup _{b_{n-1} \geqslant b_{n} \wedge \operatorname{Su}(f)}\left\{\left[b_{n-1} \wedge\left(\mu\left(\left\{f \geqslant b_{n-1}\right\}\right)+\left(b_{n} \wedge \operatorname{Su}(f)\right)\right)\right]+M_{n-1}^{*}\left(b_{n-1}\right)\right\} \\
\leqslant & \sup _{b_{n-1} \geqslant b_{n} \wedge \operatorname{Su}(f)}\left\{\left[b_{n-1} \wedge\left(\mu\left(\left\{f \geqslant b_{n-1}\right\}\right)+\operatorname{Su}(f)\right)\right]+M_{n-1}^{*}\left(b_{n-1}\right)\right\} \\
= & \sup _{b_{n-1} \geqslant b_{n} \wedge \operatorname{Su}(f)} N_{n-1}\left(b_{n-1}\right) .
\end{aligned}
$$

Here and subsequently,

$$
N_{k}(b):=\left[b \wedge\left(\mu(\{f \geqslant b\})+\mathrm{Su}_{+}^{n-k}(f)\right)\right]+M_{k}^{*}(b)
$$

for all $k=1, \ldots, n-1$ with the convention $M_{1}^{*}(b):=0$. By the very definition of $\operatorname{Su}_{+}^{2}(f)$, we have $b \wedge(\mu(\{f \geqslant b\})+\mathrm{Su}(f)) \leqslant b \wedge \mathrm{Su}_{+}^{2}(f)$ for $b \in Y$. Thus

$$
\begin{aligned}
N_{n-1}\left(b_{n-1}\right) \leqslant & \left(b_{n-1} \wedge \mathrm{Su}_{+}^{2}(f)\right)+\sup _{b_{n-2} \geqslant b_{n-1}}\left\{\left[\left(b_{n-2}-b_{n-1}\right) \wedge \mu\left(\left\{f \geqslant b_{n-2}\right\}\right)\right]+M_{n-2}^{*}\left(b_{n-2}\right)\right\} \\
\leqslant & \left(b_{n-1} \wedge \mathrm{Su}_{+}^{2}(f)\right) \\
& +\sup _{b_{n-2} \geqslant b_{n-1} \wedge \mathrm{Su}_{+}^{2}(f)}\left\{\left[\left(b_{n-2}-\left(b_{n-1} \wedge \mathrm{Su}_{+}^{2}(f)\right)\right) \wedge \mu\left(\left\{f \geqslant b_{n-2}\right\}\right)\right]+M_{n-2}^{*}\left(b_{n-2}\right)\right\} \\
= & \sup _{b_{n-2} \geqslant b_{n-1} \wedge \mathrm{Su}_{+}^{2}(f)}\left\{\left[b_{n-2} \wedge\left(\mu\left(\left\{f \geqslant b_{n-2}\right\}\right)+\left(b_{n-1} \wedge \mathrm{Su}_{+}^{2}(f)\right)\right)\right]+M_{n-2}^{*}\left(b_{n-2}\right)\right\} \\
\leqslant & \sup _{b_{n-2} \geqslant b_{n-1} \wedge \mathrm{Su}_{+}^{2}(f)}\left\{\left[b_{n-2} \wedge\left(\mu\left(\left\{f \geqslant b_{n-2}\right\}\right)+\mathrm{Su}_{+}^{2}(f)\right)\right]+M_{n-2}^{*}\left(b_{n-2}\right)\right\} \\
= & \sup _{b_{n-2} \geqslant b_{n-1} \wedge \mathrm{Su}_{+}^{2}(f)} N_{n-2}\left(b_{n-2}\right)
\end{aligned}
$$

for any $b_{n-1} \geqslant b_{n} \wedge \mathrm{Su}(f)$. In the same manner we obtain for $k=1, \ldots, n-2$,

$$
\sup _{b_{n-k} \geqslant b_{n-k+1} \wedge \mathrm{Su}_{+}^{k}(f)} N_{n-k}\left(b_{n-k}\right) \leqslant \sup _{b_{n-k-1} \geqslant b_{n-k} \wedge \mathrm{Su}_{+}^{k+1}(f)} N_{n-k-1}\left(b_{n-k-1}\right) .
$$

As a consequence, we get

$$
M_{n}\left(b_{n}, b_{n+1}\right) \leqslant \sup _{b_{n-1} \geqslant b_{n} \wedge \operatorname{Su}(f)} N_{n-1}\left(b_{n-1}\right) \leqslant \ldots \leqslant \sup _{b_{1} \geqslant b_{2} \wedge \mathrm{Su}_{+}^{n-1}(f)} N_{1}\left(b_{1}\right)=\operatorname{Su}_{+}^{n}(f)
$$

for all $b_{n} \in Y$. Therefore,

$$
\mathrm{I}_{n}^{+, \wedge}(\mu, f) \leqslant \mathrm{Su}_{+}^{n}(f)
$$


To finish the proof it is sufficient to show that $\sup _{b_{n} \in Y} M_{n}\left(b_{n}, b_{n+1}\right) \geqslant \mathrm{Su}_{+}^{n}(f)$. Using Lemma 2.1(c) and mimicking the proof for $n=2$, we obtain

$$
\begin{aligned}
\sup _{b_{n} \in Y} M_{n}\left(b_{n}, b_{n+1}\right) & \geqslant \lim _{b_{n}} M_{\operatorname{Su}(f)} M_{n}\left(b_{n}, b_{n+1}\right) \\
& =\operatorname{Su}(f)+\lim _{b_{n} \nearrow \operatorname{Su}(f)} \sup _{b_{n-1} \geqslant b_{n}}\left\{\left[\left(b_{n-1}-b_{n}\right) \wedge \mu\left(\left\{f \geqslant b_{n-1}\right\}\right)\right]+M_{n-1}^{*}\left(b_{n-1}\right)\right\} \\
& \geqslant \operatorname{Su}(f)+\lim _{b_{n} \nearrow \operatorname{Su}(f)} \sup _{b_{n-1} \geqslant b_{n}}\left\{\left[\left(b_{n-1}-\operatorname{Su}(f)\right)_{+} \wedge \mu\left(\left\{f \geqslant b_{n-1}\right\}\right)\right]+M_{n-1}^{*}\left(b_{n-1}\right)\right\} \\
& =\operatorname{Su}(f)+\sup _{b_{n-1} \geqslant \operatorname{Su}(f)}\left\{\left[\left(b_{n-1}-\operatorname{Su}(f)\right) \wedge \mu\left(\left\{f \geqslant b_{n-1}\right\}\right)\right]+M_{n-1}^{*}\left(b_{n-1}\right)\right\} \\
& =\sup _{b_{n-1} \geqslant \operatorname{Su}(f)} N_{n-1}\left(b_{n-1}\right) .
\end{aligned}
$$

By Proposition 4.4. $\mathrm{Su}_{+}^{2}(f) \geqslant \mathrm{Su}(f)>0$. Proposition 4.3 (b) and 21) implies

$$
\begin{aligned}
\sup _{b_{n} \in Y} M_{n}\left(b_{n}, b_{n+1}\right) & \geqslant \lim _{b_{n-1} \nearrow \operatorname{Su}_{+}^{2}(f)}\left(\left[b_{n-1} \wedge\left(\mu\left(\left\{f \geqslant b_{n-1}\right\}\right)+\operatorname{Su}(f)\right)\right]+M_{n-1}^{*}\left(b_{n-1}\right)\right) \\
& =\operatorname{Su}_{+}^{2}(f)+\lim _{b_{n-1} / \mathrm{Su}_{+}^{2}(f)} \sup _{b_{n-2} \geqslant b_{n-1}} M_{n-2}\left(b_{n-2}, b_{n-1}\right) .
\end{aligned}
$$

Next, we get

$$
\begin{aligned}
\lim _{b_{n-1}} \mathrm{Su}_{+}^{2}(f) & \sup _{b_{n-2} \geqslant b_{n-1}} M_{n-2}\left(b_{n-2}, b_{n-1}\right) \\
& \geqslant \lim _{b_{n-1} / \mathrm{Su}_{+}^{2}(f)} \sup _{b_{n-2} \geqslant b_{n-1}}\left\{\left[\left(b_{n-2}-\mathrm{Su}_{+}^{2}(f)\right)_{+} \wedge \mu\left(\left\{f \geqslant b_{n-2}\right\}\right)\right]+M_{n-2}^{*}\left(b_{n-2}\right)\right\} \\
& \geqslant \sup _{b_{n-2} \geqslant \mathrm{Su}_{+}^{2}(f)}\left\{\left[\left(b_{n-2}-\mathrm{Su}_{+}^{2}(f)\right) \wedge \mu\left(\left\{f \geqslant b_{n-2}\right\}\right)\right]+M_{n-2}^{*}\left(b_{n-2}\right)\right\} .
\end{aligned}
$$

Thus from 22 and 23 we obtain

$$
\sup _{b_{n} \in Y} M_{n}\left(b_{n}, b_{n+1}\right) \geqslant \sup _{b_{n-2} \geqslant \operatorname{Su}_{+}^{2}(f)} N_{n-2}\left(b_{n-2}\right) .
$$

Repeating the same reasoning we get

$$
\sup _{b_{n} \in Y} M_{n}\left(b_{n}, b_{n+1}\right) \geqslant \sup _{b_{1} \geqslant \operatorname{Su}_{+}^{n-1}(f)} N_{1}\left(b_{1}\right)=\operatorname{Su}_{+}^{n}(f),
$$

as required. The proof is complete.

Proof of Theorem 4.12. Let $n \geqslant 2$. We need to show that $\mathrm{Su}_{+}^{n}(f)=L_{n}$, where

$$
L_{n}=\inf \left\{\sum_{i=1}^{n}\left(\left(b_{i}-b_{i+1}\right) \vee \mu\left(\left\{f>b_{i}\right\}\right)\right): 0=b_{n+1} \leqslant b_{n} \leqslant \ldots \leqslant b_{1} \leqslant \bar{y}\right\} .
$$

Clearly, $L_{n}=\inf _{b_{n} \in Y} M_{n}\left(b_{n}, b_{n+1}\right)$, where $M_{n}$ is defined recursively using the formula

$$
M_{k}\left(b_{k}, b_{k+1}\right):=\left[\left(b_{k}-b_{k+1}\right) \vee \mu\left(\left\{f>b_{k}\right\}\right)\right]+\inf _{b_{k-1} \geqslant b_{k}} M_{k-1}\left(b_{k-1}, b_{k}\right)
$$


for any $k=2, \ldots, n$ with $M_{1}\left(b_{1}, b_{2}\right):=\left(b_{1}-b_{2}\right) \vee \mu\left(\left\{f>b_{1}\right\}\right)$. For simplicity, put $M_{k}^{*}\left(b_{k}\right):=$ $\inf _{b_{k-1} \geqslant b_{k}} M_{k-1}\left(b_{k-1}, b_{k}\right)$ for $k=2, \ldots, n$. Since $b \vee \mu(\{f>b\}) \geqslant \operatorname{Su}(f)$, we have $b \vee \mu(\{f>b\}) \geqslant b \vee \operatorname{Su}(f)$ for all $b \in Y$. Thus,

$$
\begin{aligned}
M_{n}\left(b_{n}, b_{n+1}\right) & \geqslant\left(b_{n} \vee \operatorname{Su}(f)\right)+\inf _{b_{n-1} \geqslant b_{n} \wedge \operatorname{Su}(f)}\left\{\left[\left(b_{n-1}-\left(b_{n} \vee \operatorname{Su}(f)\right)\right) \vee \mu\left(\left\{f>b_{n-1}\right\}\right)\right]+M_{n-1}^{*}\left(b_{n-1}\right)\right\} \\
& =\inf _{b_{n-1} \geqslant b_{n} \wedge \operatorname{Su}(f)}\left\{\left[b_{n-1} \vee\left(\mu\left(\left\{f>b_{n-1}\right\}\right)+\left(b_{n} \vee \operatorname{Su}(f)\right)\right)\right]+M_{n-1}^{*}\left(b_{n-1}\right)\right\} \\
& \geqslant \inf _{b_{n-1} \geqslant b_{n} \wedge \operatorname{Su}(f)}\left\{\left[b_{n-1} \vee\left(\mu\left(\left\{f>b_{n-1}\right\}\right)+\operatorname{Su}(f)\right)\right]+M_{n-1}^{*}\left(b_{n-1}\right)\right\} \\
& =\inf _{b_{n-1} \geqslant b_{n} \wedge \operatorname{Su}(f)} N_{n-1}\left(b_{n-1}\right),
\end{aligned}
$$

where $N_{k}(b):=\left[b \vee\left(\mu(\{f>b\})+\mathrm{Su}_{+}^{n-k}(f)\right)\right]+M_{k}^{*}(b)$ for $k=1, \ldots, n-1$ with $M_{1}^{*}\left(b_{1}\right):=0$. Theorem 4.6 gives that $b \vee(\mu(\{f>b\})+\mathrm{Su}(f)) \geqslant b \vee \mathrm{Su}_{+}^{2}(f)$ for all $b \in Y$. Thus,

$$
\begin{aligned}
N_{n-1}\left(b_{n-1}\right) \geqslant & \left(b_{n-1} \vee \mathrm{Su}_{+}^{2}(f)\right)+\inf _{b_{n-2} \geqslant b_{n-1}}\left\{\left[\left(b_{n-2}-b_{n-1}\right) \vee \mu\left(\left\{f>b_{n-2}\right\}\right)\right]+M_{n-2}^{*}\left(b_{n-2}\right)\right\} \\
\geqslant & \left(b_{n-1} \vee \mathrm{Su}_{+}^{2}(f)\right) \\
& +\inf _{b_{n-2} \geqslant b_{n-1} \wedge \mathrm{Su}_{+}^{2}(f)}\left\{\left[\left(b_{n-2}-\left(b_{n-1} \vee \mathrm{Su}_{+}^{2}(f)\right)\right) \vee \mu\left(\left\{f>b_{n-2}\right\}\right)\right]+M_{n-2}^{*}\left(b_{n-2}\right)\right\} \\
= & \inf _{b_{n-2} \geqslant b_{n-1} \wedge \mathrm{Su}_{+}^{2}(f)}\left\{\left[b_{n-2} \vee\left(\mu\left(\left\{f>b_{n-2}\right\}\right)+\left(b_{n-1} \vee \mathrm{Su}_{+}^{2}(f)\right)\right)\right]+M_{n-2}^{*}\left(b_{n-2}\right)\right\} \\
\geqslant & \inf _{b_{n-2} \geqslant b_{n-1} \wedge \mathrm{Su}_{+}^{2}(f)}\left\{\left[b_{n-2} \vee\left(\mu\left(\left\{f>b_{n-2}\right\}\right)+\mathrm{Su}_{+}^{2}(f)\right)\right]+M_{n-2}^{*}\left(b_{n-2}\right)\right\} \\
= & \inf _{b_{n-2} \geqslant b_{n-1} \wedge \mathrm{Su}_{+}^{2}(f)} N_{n-2}\left(b_{n-2}\right)
\end{aligned}
$$

for any $b_{n-1} \geqslant b_{n} \wedge \operatorname{Su}(f)$. Analogously, for $k=1, \ldots, n-2$ we get

$$
\inf _{b_{n-k} \geqslant b_{n-k+1} \wedge \mathrm{Su}_{+}^{k}(f)} N_{n-k}\left(b_{n-k}\right) \geqslant \inf _{b_{n-k-1} \geqslant b_{n-k} \wedge \mathrm{Su}_{+}^{k+1}(f)} N_{n-k-1}\left(b_{n-k-1}\right),
$$

where we use the fact that for all $b \in Y$ and $k=2, \ldots, n-1$

$$
b \vee\left(\mu(\{f>b\})+\mathrm{Su}_{+}^{k-1}(f)\right) \geqslant b \vee \mathrm{Su}_{+}^{k}(f)
$$

(see Theorem 4.6. As a consequence of 25) and 24), we obtain

$$
\begin{aligned}
M_{n}\left(b_{n}, b_{n+1}\right) & \geqslant \inf _{b_{n-1} \geqslant b_{n} \wedge \mathrm{Su}(f)} N_{n-1}\left(b_{n-1}\right) \geqslant \ldots \geqslant \inf _{b_{1} \geqslant b_{2} \wedge \mathrm{Su}_{+}^{n-1}(f)} N_{1}\left(b_{1}\right) \\
& \geqslant \inf _{b_{1} \in Y} N_{1}\left(b_{1}\right)=\mathrm{Su}_{+}^{n}(f)
\end{aligned}
$$

for all $b_{n} \in Y$. Therefore $L_{n} \geqslant \operatorname{Su}_{+}^{n}(f)$. We show that $L_{n} \leqslant \mathrm{Su}_{+}^{n}(f)$.

Let $\mathrm{Su}_{+}^{n}(f)=\bar{y}$. Then

$$
L_{n} \leqslant M_{n}\left(\bar{y}, b_{n+1}\right)=\bar{y}+M_{n}^{*}(\bar{y})=\bar{y}=\operatorname{Su}_{+}^{n}(f) .
$$


Assume that $\mathrm{Su}_{+}^{n}(f)<\bar{y}$. Using Lemma 2.1(d), we have

$$
\begin{aligned}
& L_{n} \leqslant \lim _{b_{n} \searrow \operatorname{Su}(f)} M_{n}\left(b_{n}, b_{n+1}\right) \\
& =\operatorname{Su}(f)+\lim _{b_{n} \backslash \mathrm{Su}(f)} \inf _{b_{n-1} \geqslant b_{n}}\left\{\left[\left(b_{n-1}-b_{n}\right) \vee \mu\left(\left\{f>b_{n-1}\right\}\right)\right]+M_{n-1}^{*}\left(b_{n-1}\right)\right\} \\
& \leqslant \mathrm{Su}(f)+\lim _{b_{n} \backslash \mathrm{Su}(f)} \inf _{b_{n-1} \geqslant b_{n}}\left\{\left[\left(b_{n-1}-\mathrm{Su}(f)\right) \vee \mu\left(\left\{f>b_{n-1}\right\}\right)\right]+M_{n-1}^{*}\left(b_{n-1}\right)\right\} \\
& =\operatorname{Su}(f)+\inf _{b_{n-1} \geqslant \operatorname{Su}(f)}\left\{\left[\left(b_{n-1}-\operatorname{Su}(f)\right) \vee \mu\left(\left\{f>b_{n-1}\right\}\right)\right]+M_{n-1}^{*}\left(b_{n-1}\right)\right\} \\
& =\inf _{b_{n-1} \geqslant \operatorname{Su}(f)} N_{n-1}\left(b_{n-1}\right) \text {. }
\end{aligned}
$$

From (26), Propositions 4.4 and 4.3 (c) we get

$$
\begin{aligned}
L_{n} & \leqslant \inf _{b_{n-1} \geqslant \operatorname{Su}(f)} N_{n-1}\left(b_{n-1}\right) \leqslant \inf _{b_{n-1} \geqslant \mathrm{Su}_{+}^{2}(f)} N_{n-1}\left(b_{n-1}\right) \\
& \leqslant \lim _{b_{n-1} \backslash \mathrm{Su}_{+}^{2}(f)}\left(\left[b_{n-1} \vee\left(\mu\left(\left\{f>b_{n-1}\right\}\right)+\mathrm{Su}(f)\right)\right]+M_{n-1}^{*}\left(b_{n-1}\right)\right) \\
& =\mathrm{Su}_{+}^{2}(f)+\lim _{b_{n-1} \backslash \mathrm{Su}_{+}^{2}(f)} M_{n-1}^{*}\left(b_{n-1}\right) .
\end{aligned}
$$

Next, we have

$$
\begin{aligned}
\lim _{b_{n-1} \searrow \mathrm{Su}_{+}^{2}(f)} M_{n-1}^{*}\left(b_{n-1}\right) & \leqslant \lim _{b_{n-1} \searrow \mathrm{Su}_{+}^{2}(f)} \inf _{b_{n-2} \geqslant b_{n-1}}\left\{\left[\left(b_{n-2}-\mathrm{Su}_{+}^{2}(f)\right) \vee \mu\left(\left\{f>b_{n-2}\right\}\right)\right]+M_{n-2}^{*}\left(b_{n-2}\right)\right\} \\
& \leqslant \inf _{b_{n-2} \geqslant \mathrm{Su}_{+}^{2}(f)}\left\{\left[\left(b_{n-2}-\mathrm{Su}_{+}^{2}(f)\right) \vee \mu\left(\left\{f>b_{n-2}\right\}\right)\right]+M_{n-2}^{*}\left(b_{n-2}\right)\right\} .
\end{aligned}
$$

Thus, from (27) and 28) we obtain

$$
L_{n} \leqslant \inf _{b_{n-1} \geqslant \mathrm{Su}_{+}^{2}(f)} N_{n-2}\left(b_{n-2}\right) .
$$

Repeating the same reasoning we get

$$
L_{n} \leqslant \inf _{b_{1} \geqslant \operatorname{Su}_{+}^{n-1}(f)} N_{1}\left(b_{1}\right)=\operatorname{Su}_{+}^{n-1}(f)+\inf _{b \geqslant \operatorname{Su}_{+}^{n-1}(f)}\left\{\left(b-\operatorname{Su}_{+}^{n-1}(f)\right) \vee \mu(\{f>b\})\right\}=\operatorname{Su}_{+}^{n}(f),
$$

as required.

\section{References}

[1] Bassan, B., \& Spizzichino, F. (2005). Relations among univariate aging, bivariate aging and dependence for exchangeable lifetimes. Journal of Multivariate Analysis, 93, 313-339.

[2] Beliakov, G., Bustince, H., \& Calvo, T. (2016). A practical guide to averaging functions. (1st ed.). Heidelberg: Springer. 
[3] Benvenuti, P., Mesiar, R., \& Vivona, D. (2002). Monotone set functions-based integrals. In E. Pap (Ed.), Handbook of Measure Theory (pp. 1329-1379). Amsterdam: Elsevier.

[4] Béal, S., Ferrières, S., Rémila, E., \& Solal, P. (2016). An axiomatization of the iterated $h$-index and applications to sport rankings. Working papers, 2016-11, CRESE.

[5] Boczek, M., \& Kaluszka, M. (2016). On the Minkowski-Hölder type inequalities for generalized Sugeno integrals with an application. Kybernetika, 52, 329-347.

[6] Boczek, M., \& Kaluszka, M. (2017). On conditions under which some generalized Sugeno integrals coincide: A solution to Dubois' problem. Fuzzy Sets and Systems, 326, 81-88.

[7] Borzová-Molnárová, J., Halčinová, L., \& Hutník, O. (2015). The smallest semicopula-based universal integrals I: Properties and characterizations. Fuzzy Sets and Systems, 271, 1-17.

[8] Brandão, L.C., \& de Mello, J.C.C.B.S. (2019). A multi-criteria approach to the $h$-index. European Journal of Operational Research, 276, 357-363.

[9] Bustince, H., Fernández, J., Kolesárová, A., \& Mesiar, R. (2015). Directional monotonicity of fusion functions. European Journal of Operational Research, 244, 300-308.

[10] Chateauneuf, A., Grabisch, M., \& Rico, A. (2008). Modeling attitudes toward uncertainty through the use of the Sugeno integral. Journal of Mathematical Economics, 44, 1084-1099.

[11] Choquet, G. (1954). Theory of capacities. Annales de l'institut Fourier, 5, 131-295.

[12] Couceiro, M., Dubois, D., Prade, H., \& Waldhauser, T. (2016). Decision-making with Sugeno integrals. Order, 33, 517-535.

[13] Deineko, W.G., \& Woeginger, G.J. (2009). A new family of scientific impact measures: The generalized Kosmulski-indices. Scientometrics, 80, 819-826.

[14] Dubois, D., Prade, H., Rico, A., \& Teheux, B. (2017). Generalized Qualitative Sugeno Integrals. Information Sciences, 415-416, 429-445.

[15] Durante, F., \& Sempi, C. (2005). Semicopulæ. Kybernetika, 41, 315-328.

[16] Durante, F., \& Sempi, C. (2016). Principles of copula theory. (1st ed.). Boca Raton: CRC Press.

[17] Franceschini, F., \& Maisano, D.A. (2010). Analysis of the Hirsch index's operational properties. European Journal of Operational Research, 203, 494-504. 
[18] García-Pérez, M. (2009). A multidimensional extension to Hirsch's h-index. Scientometrics, 81, $779-785$.

[19] García-Pérez, M. (2012). An extension of the $h$ index that covers the tail and the top of the citation curve and allows ranking researchers with similar $h$. Journal of Informetrics, 6, 689-699.

[20] Gągolewski, M., \& Mesiar, R. (2012). Aggregating different paper quality measures with a generalized $h$-index. Journal of Informetrics, 6, 566-579.

[21] Gągolewski, M., \& Mesiar, R. (2014). Monotone measures and universal integrals in a uniform framework for the scientific impact assessment problem. Information Sciences, 263, 166-174.

[22] Greco, S., Mesiar, R., Rindone, F., \& Sipeky, L. (2016). Decomposition approaches to integration without a measure. Fuzzy Sets and Systems, 287, 37-47.

[23] Hirsch, J.E. (2005). An index to quantify an individual's scientific research output. Proceedings of the National Academy of Sciences of USA, 102, 16569-16572.

[24] Jin, L., Mesiar, R., Stupňanová, A.: Sugeno integrals, $H_{\alpha}$ and $H^{\beta}$ indices: how to compare scientists from different academic areas. IEEE Transactions on Fuzzy Systems. DOI 10.1109/TFUZZ.2019.2914625.

[25] Kaluszka, M., Okolewski, A., \& Boczek, M. (2014). On Chebyshev type inequalities for generalized Sugeno integrals. Fuzzy Sets and Systems, 244, 51-62.

[26] Kawabe, J. (2015). The bounded convergence in measure theorem for nonlinear integral functionals. Fuzzy Sets and Systems, 271, 31-42.

[27] Kosmulski, M. (2006). A new Hirsch-index saves time and works equally well as the origin $h$-index. ISSSI Newsletter, 2, 4-6.

[28] Mesiar, R., \& Gągolewski, M. (2016). H-index and other Sugeno integrals: some defects and their compensation. IEEE Transactions on Fuzzy Systems, 24, 1668-1672.

[29] Mesiar, R., Kolesárová, A., Bustince, H., Dimuro, G.P., \& Bedregal, B.C. (2016). Fusion functions based discrete Choquet-like integrals. European Journal of Operational Research, 252, 601-609.

[30] Mesiar, R., \& Stupňanová, A. (2013). Decomposition integrals. International Journal of Approximate Reasoning, 54, 1252-1259.

[31] Mingers, J., \& Leydesdorff, J. (2015). A review of theory and practice in scientometrics. European Journal of Operational Research, 246, 1-19. 
[32] Miroiu, A. (2013). Axiomatizing the Hirsch index: Quantity and quality disjoined. Journal of Informetrics, 7, 10-15.

[33] Quesada, A. (2011). Further characterizations of the Hirsch index. Scientometrics, 87, 107-114.

[34] Rousseau, R. (2006). New developments related to the Hirsch index. Science Focus, 1, 23-25.

[35] Struk, P. (2006). Extremal fuzzy integrals. Soft Computing, 10, 502-505.

[36] Suárez García, F., \& Gil Álvarez, P. (1986). Two families of fuzzy integrals. Fuzzy Sets and Systems, 18, 67-81.

[37] Sugeno, M. (1974). Theory of Fuzzy Integrals and Its Applications. Tokyo Institute of Technology, Tokyo. Ph.D. thesis.

[38] Torra, V., \& Narukawa, Y. (2006). The interpretation of fuzzy integrals and their application to fuzzy systems. International Journal of Approximate Reasoning, 41, 43-58.

[39] Torra, V., \& Narukawa, Y. (2008). The $h$-index and the number of citations: Two fuzzy integrals. IEEE Transactions on Fuzzy Systems, 16, 795-797.

[40] Van Eck, N.J., \& Waltman, L. (2008). Generalizing the $h$-and $g$-indices. Journal of Informetrics, 2, 263-271.

[41] Wang, Z., \& Klir, G. (2009). Generalized Measure Theory. (1st ed.). New York: Springer.

[42] Woeginger, G.J. (2008). An axiomatic characterization of the Hirsch-index. Mathematical Social Sciences, 56, 224-232.

[43] Woeginger, G.J. (2008). A symmetry axiom for scientific impact indices. Journal of Informetrics, 2, 298-303.

[44] Zhai, J., Zang, L., \& Zhou, Z. (2018). Ensemble dropout extreme learning machine via fuzzy integral for data classification. Neurocomputing, 275, 1043-1052. 